\title{
Causal correlation functions and Fourier transforms: Application in calculating pressure induced shifts
}

\author{
Q. Ma ${ }^{\mathrm{a}, *}$, R.H. Tipping ${ }^{\mathrm{b}, 1}$, N.N. Lavrentieva ${ }^{\mathrm{c}, 2}$ \\ a NASA/Goddard Institute for Space Studies and Department of Applied Physics and Applied Mathematics, Columbia University, 2880 Broadway, \\ New York, NY 10025, USA \\ ${ }^{\mathrm{b}}$ Department of Physics and Astronomy, University of Alabama, Tuscaloosa, AL 35487-0324, USA \\ ${ }^{\mathrm{c}}$ V.E. Zuev Institute of Atmospheric Optics SB RAS, 1, Akademician Zuev square, Tomsk 634021, Russia
}

\section{A R T I C L E I N F O}

\section{Article history:}

Received 10 November 2011

Received in revised form

3 February 2012

Accepted 4 February 2012

Available online 18 February 2012

\section{Keywords:}

Correlation function

Causal function

Fourier transform and Hilbert transform

Pressure broadened half-widths

Pressure induced shifts

Spectral lines of $\mathrm{H}_{2} \mathrm{O}$

HITRAN

\begin{abstract}
A B S T R A C T
By adopting a concept from signal processing, instead of starting from the correlation functions which are even, one considers the causal correlation functions whose Fourier transforms become complex. Their real and imaginary parts multiplied by 2 are the Fourier transforms of the original correlations and the subsequent Hilbert transforms, respectively. Thus, by taking this step one can complete the two previously needed transforms. However, to obviate performing the Cauchy principal integrations required in the Hilbert transforms is the greatest advantage. Meanwhile, because the causal correlations are well-bounded within the time domain and band limited in the frequency domain, one can replace their Fourier transforms by the discrete Fourier transforms and the latter can be carried out with the FFT algorithm. This replacement is justified by sampling theory because the Fourier transforms can be derived from the discrete Fourier transforms with the Nyquis rate without any distortions. We apply this method in calculating pressure induced shifts of $\mathrm{H}_{2} \mathrm{O}$ lines and obtain more reliable values. By comparing the calculated shifts with those in HITRAN 2008 and by screening both of them with the pair identity and the smooth variation rules, one can conclude many of shift values in HITRAN are not correct.
\end{abstract}

(c) 2012 Elsevier Ltd. All rights reserved.

\section{Introduction}

In order to carry out forward modeling of atmospheric radiative transfer processes and to obtain information on the abundances of molecular species, temperature-pressure profiles, and other atmospheric properties, one needs accurate spectroscopic data. This includes not only line positions, strengths, and the temperature-dependent Lorentzian halfwidths, but also pressure induced shifts. Because the

\footnotetext{
* Corresponding author. Tel.: +1 212678 5574; fax: +1 2126785552. E-mail addresses: qiancheng.ma@nasa.gov (Q. Ma), rtipping@bama.ua.edu (R.H. Tipping), Inn@iao.ru (N.N. Lavrentieva).

${ }^{1}$ Tel.: +1 205248 3799; fax: +1 2053485051 .

${ }^{2}$ Tel.: +7 3822490 067; fax: +7 3822492086 .
}

ambient atmospheric species, temperatures, and pressures are not always amenable to laboratory measurements, or because of the large number of transitions possible, one often has to rely on theoretical calculations. This is especially true for the induced shift because their values are smaller than values of the half-width so that to perform reliable laboratory measurements becomes more difficult.

It is well known that there are formalisms available for many years such as the Anderson-Tsao-Curnutte (ATC) theory $[1,2]$, the Robert-Bonamy (RB) theory [3,4] and others with which one can calculate half-widths and shifts of molecular spectral lines. These formalisms are based on two basic approximations: the binary collision and the impact approximations. With these two approximations, calculations of the pressure broadened halfwidths and induced shifts of the absorber molecular lines 
are reduced to thermal averages of the "connected" operator $M_{c}(\omega)$ introduced by Fano over all possible collision processes involving a pair of molecules [5], and in comparison with the time of interest, these collisions are assumed to be completed instantaneously. In addition, both the ATC and RB formalism are semi-classical theories. In other words, the translational motion of the pair is treated classically, while their internal degrees of freedom are treated quantum mechanically. This semi-classical method is valid in calculating molecular spectral lines for temperatures of interest in atmospheric applications. However, the current theories are also based on other assumptions. Some of these lack sound justification and could lead to uncertainties that affect the reliability of the calculated results. Besides, it is commonly accepted by most researchers that in comparison with calculated half-widths, calculated shifts usually contain larger uncertainties. According to our knowledge, there is lack of systematic studies to explain why the theoretically calculated shifts are poorer than the calculated half-widths. One possible reason could be severe cancelations of contributions to the lineshifts occur in calculation processes. To identify other reasons requires individually investigating the entire calculation processes within the specified formalisms.

With the formalism developed from the introduction of the coordinate representation in Hilbert space, [6,7] the usual route in calculating converged half-widths and lineshifts consists of several steps. For simplicity, we omit subscripts attached to following functions. First, one calculates the irreducible correlation functions $F(t)$ of the $\hat{S}$ matrix based on the potential and trajectory models selected. Then, by carrying out the Fourier transforms of $F(t)$, one obtains functions $H(\omega)$. Next, by evaluating the Cauchy principal integrations for $H(\omega)$, one obtains $I(\omega)$, the Hilbert transforms of $H(\omega)$. After these $H(\omega)$ and $I(\omega)$ are available, one calculates the real part of $S_{2}$, the second-order of the perturbation expansion of the $\hat{S}$ matrix, from $H(\omega)$ and the imaginary part of $S_{2}$ from $I(\omega)$ for specified lines. Finally, one derives their calculated half-widths and shifts.

Following this route, the entire process goes smoothly until one has to do the Cauchy principal integrations. There are subroutines available in computer libraries with which one can carry out the Cauchy principal integrations. In general, these subroutines work well for most cases. However, it turns out their unstable performances do happen occasionally. Given the fact that in practical calculations one needs to evaluate a huge number of the Cauchy principal integrations of the order of $10^{7}$, even seldom occurring performance failures could cause severe consequences because it is not easy to identify all of them and to make remedies accordingly. As a result, our previous attempts to calculate converged results for lineshift stalled at this stage. In fact, except for reporting calculated half-widths of $\mathrm{H}_{2} \mathrm{O}$ lines [7], we have not published any calculated shifts for $\mathrm{H}_{2} \mathrm{O}$ lines because of the instability problem mentioned above. Even for our calculated half-widths, in order to avoid involving $I(\omega)$ we had to follow other researchers' assumption that the contributions from the imaginary part of $S_{2}$ can be ignored. Fortunately, thanks to the present study the validity of this assumption has been verified. Therefore, within our formalism it is the Cauchy principal integration that causes more difficulty in calculating the lineshift than the half-width. Unless we are able to find an alternative route to reach $I(\omega)$, we simply cannot provide reliable lineshift values at all. Despite realizing this problem for years, we had not found an effective solution to overcome this challenge until now.

Very recently, an idea occurred that because the Fourier and Hilbert transforms are commonly used in many science and engineering fields, why not pursue a search for tools used in other disciplines? This effort paid off quickly, and we found a useful tool that we were looking for. By adopting the concept of the causal function from signal processing, we have found that instead of starting from the usual correlations which are even functions and whose Fourier transforms are real, we can consider the causal correlations which are neither even nor odd and whose Fourier transforms become complex. But the most striking feature of the latter's Fourier transforms is that their real and imaginary parts are simply equal to $H(\omega) / 2$ and $I(\omega) / 2$, respectively. With this tool, the Cauchy principal integrations are completely obviated. Meanwhile, thanks to sampling theory, the Fourier transforms can be replaced by the discrete Fourier transforms with the Nyquist rate without any distortions [8]. Furthermore, the latter can be easily carried out with the fast Fourier transforms (FFT) [9]. Our numerical tests demonstrate accuracies achieved for $H(\omega)$ and $I(\omega)$ derived are well satisfied. Especially for $I(\omega)$, where one is able to achieve very high accuracies in large ranges of $\omega$. Thus, with this simple method, the challenge has been completely overcome.

The present manuscript is organized in the following way. In Section 2, we only outline the basic of the modified RB formalism developed in the coordinate representation [6,7], but we provide explanations in detail about the correlation functions, their Fourier transforms, and the subsequent Hilbert transforms. Section III is devoted to the new route in which we start by introducing the concept of the causal function and its Fourier transform. Then, we briefly explain the sampling theory with which one is able to replace a Fourier transform by a discrete Fourier transform. Finally, by presenting examples, we demonstrate how the new method works in evaluating the Hilbert transform without performing the Cauchy principal integrations and explicitly show how high accuracies of the new method can be achieved. In Section 4, we present our calculated shifts for $\mathrm{H}_{2} \mathrm{O}$ lines in the pure rotational band and make comparisons with those listed in HITRAN 2008, and other fruitful results from the present study are also presented. The last Section 5 consists of a brief discussions and conclusions.

\section{General formalism in calculating $\mathrm{N}_{2}$ induced shifts for $\mathrm{H}_{2} \mathrm{O}$ lines}

\subsection{The RB formalism applicable for complicated potential models}

Among all line shape theories available at present, the $\mathrm{RB}$ formalism is one of the most widely adopted. In fact, it 
is this formalism with which theoretical calculations of the half-widths and shifts of $\mathrm{H}_{2} \mathrm{O}$ lines have been carried out for years [10-13] and the results have played important roles in updating the HITRAN database [14]. We briefly outline the formalism used to calculate the halfwidths and shifts of $\mathrm{H}_{2} \mathrm{O}$ lines.

The main computational task for calculating the Lorentzian half-widths and shifts is the evaluations of matrix elements appearing in the perturbation expansion of the Liouville operator $\hat{\mathrm{S}}\left(=S_{I} \cdot S_{\mathrm{F}}^{*}\right.$, where $S_{I}$ and $S_{F}$ are scattering matrices in Hilbert space). Usually, in practice, these evaluations are limited to the second-orders of the expansion. Within the RB formalism, the original expressions for the pressure broadened half-width and induced shift are given by

$$
\begin{aligned}
\gamma_{R B}= & \frac{n_{b}}{2 \pi c} \int_{0}^{+\infty} v f(v) d v \int_{0}^{+\infty} 2 \pi b d b \\
& \times\left\langle 1-\cos \left[S_{1}(b)+\operatorname{Im} S_{2}(b)\right] e^{-\operatorname{ReS}_{2}(b)}\right\rangle_{j_{2}},
\end{aligned}
$$

and

$$
\begin{aligned}
\delta_{R B}= & \frac{n_{b}}{2 \pi c} \int_{0}^{+\infty} v f(v) d v \int_{0}^{+\infty} 2 \pi b d b \\
& \times\left\langle\sin \left[S_{1}(b)+\operatorname{Im} S_{2}(b)\right] e^{-\operatorname{Re}_{2}(b)}\right\rangle_{j_{2}} .
\end{aligned}
$$

where $n_{b}$ is the number density of the bath molecule, $f(v)$ is the Maxwell-Boltzmann distribution function, $S_{1}$ and $S_{2}$ are matrix elements in the Liouville space associated with the first- and second-orders of the perturbation expansion of the Liouville operator $\hat{S}$, and $\langle\cdots\rangle j_{2}$ means an average over the quantum number $j_{2}$ of the bath molecule. However, we have found a subtle error in the RB formalism [15]. After remedying this derivation error, the correct expression for the half-width and lineshift in the "modified" RB formalism (MRB) become [15]

$$
\begin{aligned}
\gamma_{M R B}= & \frac{n_{b}}{2 \pi c} \int_{0}^{+\infty} v f(v) d v \int_{0}^{+\infty} 2 \pi b d b \\
& \times\left\{1-\cos \left[\left\langle S_{1}(b)\right\rangle_{j_{2}}+\operatorname{Im}\left\langle S_{2}(b)\right\rangle_{j_{2}}\right] e^{-\operatorname{Re}\left\langle S_{2}(b)\right\rangle_{j_{2}}}\right\} .
\end{aligned}
$$

and

$$
\begin{aligned}
\delta_{M R B}= & \frac{n_{b}}{2 \pi c} \int_{0}^{+\infty} v f(v) d v \int_{0}^{+\infty} 2 \pi b d b \\
& \times \sin \left(\left\langle S_{1}(b)\right\rangle_{j_{2}}+\operatorname{Im}\left\langle S_{2}(b)\right\rangle_{j_{2}}\right) e^{-\operatorname{Re}\left\langle S_{2}(b)\right\rangle_{j_{2}}} .
\end{aligned}
$$

We note that the essential difference between these expressions is that in Eqs. (1) and (2) the summation over $j_{2}$ is outside of the cumulant expansion while in contrast, in Eqs. (3) and (4) it is inside. It is worthwhile emphasizing that because the bath average is carried out in the line space, an average over $j_{2}$ must be included. Thus, by understanding that $S_{1}(b)$ and $S_{2}(b)$ are the bath averages associated with the first- and second-order expansions of the Liouville operator $\hat{S}$, one can suppress all of these $\langle\cdots\rangle j_{2}$ in Eqs. (3) and (4). For simplicity, we will omit the subscript MRB of $\gamma$ and $\delta$.

Usually, people prefer to represent $S_{2}$ by three components labeled by $S_{2, \text { outer, }}\left(r_{c}\right), S_{2, \text { outer } f}\left(r_{c}\right)$, and $S_{2, \text { middle }}\left(r_{c}\right)$, respectively. In the present study, we follow the same custom. As an example, an expression for $S_{2, \text { outer,i }}\left(r_{c}\right)$ is given by $[6,7]$

$$
\begin{aligned}
& S_{2, \text { outer }, i}\left(r_{c}\right) \frac{1}{\hbar^{2}\left(2 j_{i}+1\right)} \sum_{j_{2} j_{2}^{\prime}} \rho_{j_{2}} \sum_{j_{i}^{\prime} \tau_{i}^{\prime}} \sum_{(m)} \\
&\left.\times \int_{-\infty}^{\infty} d t \int_{-\infty}^{t} d t^{\prime} e^{i\left(\omega_{j_{i}} \tau_{i}^{\prime} i_{i}^{\prime} \tau_{i}^{\prime}\right.}+\omega_{j_{2} j_{2}^{\prime}}\right)\left(t-t^{\prime}\right) \\
& \quad \times\left\langle j_{i} \tau_{i} m_{i} j_{2} m_{2}|V(\vec{R}(t))| j_{i}^{\prime} \tau_{i}^{\prime} m_{i}^{\prime} j_{2}^{\prime} m_{2}^{\prime}\right\rangle \\
& \quad \times\left\langle j_{i}^{\prime} \tau_{i}^{\prime} m_{i}^{\prime} j_{2}^{\prime} m_{2}^{\prime}\left|V\left(\vec{R}\left(t^{\prime}\right)\right)\right| j_{i} \tau_{i} m_{i} j_{2} m_{2}\right\rangle,
\end{aligned}
$$

where $\vec{R}(t)$ represents the translational motion of two interacting molecules. In the above equation, $\rho_{j_{2}}$ is the density matrix of the bath molecule, $(m)$ means summations over all magnetic quantum numbers, $\omega_{j_{2} j_{2}^{\prime}}=$ $\left[E^{(b)}\left(j_{2}\right)-E^{(b)}\left(j_{2}^{\prime}\right)\right] / \hbar$, and $\left|j_{i} \tau_{i} m_{i} j_{2} m_{2}>=\right| j_{i} \tau_{i} m_{i}>\otimes \mid j_{2} m_{2}>$ where $\mid j_{i} \tau_{i} m_{i}>$ and $\mid j_{2} m_{2}>$ are the basis of Hilbert space for the $\mathrm{H}_{2} \mathrm{O}$ and $\mathrm{N}_{2}$ molecules, respectively, and $\tau$ is a simple notation for $k_{a}-k_{c}$ in $\mid j \tau m>$. In practical calculations, how to evaluate these three $S_{2}$ terms more accurately presents a big challenge.

In order to evaluate the potential matrix elements in Eq. (5), one prefers to express $V(\vec{R}(t))$ in terms of the standard spherical expansions

$$
\begin{aligned}
& V(\vec{R}(t))=\sum_{L_{1} K_{1} L_{2} L} u\left(L_{1} L_{2} L ; K_{1} ; R(t)\right) \\
& \quad \times \sum_{m_{1} m_{2} m} C\left(L_{1} L_{2} L, m_{1} m_{2} m\right) D_{m_{1} K_{1}}^{L_{1} *}\left(\Omega_{a}\right) D_{m_{2} 0}^{L_{2} *}\left(\Omega_{b}\right) Y_{L m}^{*}(\omega(t)),
\end{aligned}
$$

where $\vec{R}(t)$ is described by its polar coordinates $R(t)$ and $\omega(t)$, and $\Omega_{a}$ and $\Omega_{b}$ are orientations of the absorber and bath molecules, respectively. In the present study, we adopt potential models for the $\mathrm{H}_{2} \mathrm{O}$ and $\mathrm{N}_{2}$ molecules consisting of long-range dipole-quadrupole and quadrupolequadrupole interactions $V_{d q}\left(\Omega_{a}, \Omega_{b}, \vec{R}\right)+V_{q q}\left(\Omega_{a}, \Omega_{b}, \vec{R}\right)$ and a short-range interaction $V_{\text {atom-atom }}\left(\Omega_{a}, \Omega_{b}, \vec{R}\right)$ modeled by the site-site model. For $V_{d q}$ and $V_{q q}$, their spherical expansions are well known and numbers of their expansion terms are very limited. For $V_{d q}$, the expansion contains only one term (i.e., $L_{1}=1, K_{1}=0, L_{2}=2$, and $L=3$ ) which varies with $R$ as $R^{-4}$. Meanwhile, for $V_{q q}$ it has three terms (i.e., $L_{1}=2, K_{1}=0, \pm 2, L_{2}=2$, and $L=4$ ) which vary as $R^{-5}$

With respect to $V_{\text {atom-atom }}$, the expression for the sitesite model is given by

$V_{\text {atom-atom }}\left(\Omega_{a}, \Omega_{b}, \vec{R}\right)=\sum_{i \in a} \sum_{j \in b} 4 \varepsilon_{i j}\left\{\frac{\sigma_{i j}^{12}}{r_{i j}^{12}}-\frac{\sigma_{i j}^{6}}{r_{i j}^{6}}\right\}$,

where $\sigma_{i j}$ and $\varepsilon_{i j}$ are parameters and $r_{i j}$ are distances between the $i$-th atom of the absorber molecule a (i.e., $\mathrm{H}_{2} \mathrm{O}$ ) and the $j$-th atom of the bath molecule b (i.e., $\mathrm{N}_{2}$ ). In order to evaluate its matrix elements, one needs to rewrite $V_{\text {atom-atom }}$ in terms of the standard spherical expansions

$$
\begin{aligned}
V_{\text {atom-atom }}\left(\Omega_{a}, \Omega_{b}, \vec{R}(t)\right) \\
=\sum_{L_{1} K_{1} L_{2} L} \sum_{n_{\{i j\}}} \sum_{w q} \frac{U\left(L_{1} K_{1} L_{2} L, n_{\{i j\}}, w q\right)}{R^{L_{1}+L_{2}+q+2 w}(t)} \\
\quad \times \sum_{m_{1} m_{2} m} C\left(L_{1} L_{2} L, m_{1} m_{2} m\right) D_{m_{1} K_{1}}^{L_{1} *}\left(\Omega_{a}\right) D_{m_{2} 0}^{L_{2} *}\left(\Omega_{b}\right) Y_{L m}^{*}(\omega(t)),
\end{aligned}
$$


where $n_{\{i j\}}$ runs over all pairs of atoms in Eq. (7), $q=6$ or $12, w$ is an integer index from 0 to infinity, and the definitions for $U\left(L_{1} K_{1} L_{2} L, n_{\{i j\}}, w q\right)$ can be found in the literature [16].

As shown in Eq. (8), when one evaluates the matrix elements of $V_{\text {atom-atom }}(t)$, there would be a lot of summation indices. In order to evaluate $S_{2, \text { outer, } i}\left(r_{c}\right)$, one needs to consider the products of two potential matrix elements. Thus, the total number of summation terms in Eq. (5) becomes so large that the evaluations are intractable unless cut-offs are enforced. It turns out that there are two kinds of cut-offs associated with the summation indices in Eqs. (8) [6,7]. The first is a cut-off to limit the set of irreducible tensor ranks $L_{1}$ and $L_{2}$ and the second is to set an upper limit for the index $w$. In the literature, if one chooses 8 as the maximum of $2 w$, the cut-off is said to be the 8-th order. It is the introduction of these two cut-offs that opens the possibility that the results derived are not converged. We have provided in detail analyses on the convergence problems existing in this method and we do not repeat them here. Readers interested in this subject are referred to our previous works [6,7].

\subsection{Irreducible correlation functions of the $\hat{S}$ matrix in the coordinate representation}

In order to overcome the convergence problem, we introduce the coordinate representation [6] whose basic idea is briefly outlined here. It is well known from quantum mechanics that one has the freedom to choose different representations in Hilbert space. The state representation that is commonly used consists of the basis set $\mid j_{i} \tau_{i} m_{i}>\otimes$ $\left|j_{2} m_{2}\right\rangle$ which are eigenfunctions of the Hamiltonian of the molecular pair. On the other hand, one can select the orientations of the pair of molecules as the basis set in Hilbert space; i.e., $\left.\left|\delta\left(\Omega_{a}-\Omega_{a \alpha}\right)>\otimes\right| \delta\left(\Omega_{b}-\Omega_{b \alpha}\right)\right\rangle$ where $\Omega_{a \alpha}$ and $\Omega_{b \alpha}$ represent orientations of $\mathrm{H}_{2} \mathrm{O}$ and $\mathrm{N}_{2}$ specified by $\alpha$, respectively. By choosing the coordinate representation, the potential becomes a diagonal operator and can be treated as classical functions [6,7]. Then, by introducing the correlation functions described later, one is able to calculate the half-widths with high accuracy, no matter how complicated the potential models are and no matter what kind of trajectories are chosen. In any case, the convergence of the calculated results is always guaranteed.

We will not provide a detailed development of the new formalism here and readers can find its derivations from our previous works [6,7]. We only present some important formulas here. The correlation functions are defined by

$F_{L_{1} K_{1} K_{1}^{\prime} L_{2}}(t)=\int_{-\infty}^{\infty} d t^{\prime} G_{L_{1} K_{1} K_{1}^{\prime} L_{2}}\left(t^{\prime}+t / 2, t^{\prime}-t / 2\right)$,

where $G_{L_{1} K_{1} K_{1}^{\prime} L_{2}}\left(t, t^{\prime}\right)$ is given by

$$
\begin{aligned}
G_{L_{1} K_{1} K_{1}^{\prime} L_{2}}\left(t, t^{\prime}\right)= & \frac{1}{4 \pi \hbar^{2}\left(2 L_{1}+1\right)^{2}\left(2 L_{2}+1\right)^{2}} \\
& \times(-1)^{K_{1}+K_{1}^{\prime}} \sum_{L}(-1)^{\left(L_{1}+L_{2}+L\right)}(2 L+1) \\
& \times u\left(L_{1} L_{2} L ; K_{1} ; R(t)\right) u\left(L_{1} L_{2} L ; K_{1}^{\prime} ; R\left(t^{\prime}\right)\right) P_{L}\left(\cos \Theta_{t t^{\prime}}\right) .
\end{aligned}
$$

In Eq. (10), the factor $(-1)^{\left(L_{1}+L_{2}+L\right)}=1$ because the summation index $L$ must satisfy $L_{1}+L_{2}+L=$ even, and $\Theta_{t t^{\prime}}$ are angles between two vectors $\vec{R}(t)$ and $\vec{R}\left(t^{\prime}\right)$. The set of indices used to label the correlation functions consist of one tensor rank $L_{1}$ with two subsidiary indices $K_{1}, K_{1}{ }^{\prime}$ related to $\mathrm{H}_{2} \mathrm{O}$ and another tensor rank $L_{2}$ for $\mathrm{N}_{2}$. Because $\mathrm{N}_{2}$ is a diatomic molecule, $L_{2}$ must be even. If one chooses the II $R$ representation to develop the $\mathrm{H}_{2} \mathrm{O}$ wave functions where two $\mathrm{H}$ atoms are symmetrically located in the molecularfixed frame, values of $K_{1}$ and $K_{1}{ }^{\prime}$ must also be even.

In the standard method, the functions required to be evaluated are the resonance functions and their total number depends on how many combination choices of summation indices $L_{1}, K_{1}, L_{2}, L$, and $q, n_{(i j)}$, w are used in Eq. (8) with the restrictions enforced by the two kinds of cut-offs. As these two cut-offs increase, the number of resonance functions increases very quickly and to evaluate all these resonance functions become formidable. On the other hand, with the new method, the functions required to be evaluated are the correlation functions and their total number is determined only by the upper limits of $L_{1}$ and $L_{2}$ restricted by the first kind of cut-off [6,7]. This means that no matter how high the second kind of cut-off is, the number of correlation functions remains unchanged. In addition, because $V_{\text {atom-atom }}$ becomes ordinary functions, one can choose any order cut-off one wants. No matter how high this cut-off order goes, there are only small differences determined by the needed computational resources. As a result, we can use both cut-offs which are sufficiently high to guarantee full convergence in practical calculations. It is easy to show that if one sets 2 as the maxima for both $L_{1}$ and $L_{2}$, the number of correlations is 20 . Meanwhile, if one increases the maximum for $L_{1}$ from 2 to 3 or 4 , the number of correlations increases from 20 to 38 or 88 , respectively. Finally, by setting 4 as the maxima for both $L_{1}$ and $L_{2}$, the number becomes 132 .

Then, in terms of the correlations, one is able to rewrite Eq. (5) as

$S_{2, \text { outer }, i}\left(r_{c}\right)=\sum_{L_{1} K_{1} K_{1}^{\prime} L_{2}} \int_{0}^{\infty} d t W_{L_{1} K_{1} K_{1}^{\prime}}^{(a)}(t) W_{L_{2}}^{(b)}(t) F_{L_{1} K_{1} K_{1}^{\prime} L_{2}}(t)$.

In deriving the above expression, two functions which are independent of the potential and trajectory models are defined by

$$
\begin{aligned}
W_{L_{1} K_{1} K_{1}^{\prime}}^{(a)}(t)= & \sum_{j_{i}^{\prime} \tau_{i}^{\prime}}\left(2 j_{i}^{\prime}+1\right) D\left(j_{i} \tau_{i} j_{i}^{\prime} \tau_{i}^{\prime} ; L_{1} K_{1}\right) \\
& \times D\left(j_{i} \tau_{i} j_{i}^{\prime} \tau_{i}^{\prime} ; L_{1} K_{1}^{\prime}\right) e^{i \omega_{j_{i}} \tau_{i}^{\prime} j_{i}^{\prime} \tau_{i}^{t}},
\end{aligned}
$$

where $D\left(j \tau j^{\prime} \tau^{\prime} ; L K\right)$ is defined by

$D\left(j \tau j^{\prime} \tau^{\prime} ; L K\right) \equiv \sum_{k}(-1)^{k} U_{k \tau}^{j} U_{k-K \tau^{\prime}}^{j,} C\left(j j^{\prime} L, k K-k K\right)$,

and

$W_{L_{2}}^{(b)}(t)=\sum_{j_{2} j_{2}^{\prime}}\left(2 j_{2}+1\right)\left(2 j_{2}^{\prime}+1\right) \rho_{j_{2}} C^{2}\left(j_{2} j_{2}^{\prime} L_{2}, 000\right) e^{i \omega_{j_{2} j_{2}}{ }^{t}}$,

respectively. In Eq. (13), $U_{k \tau}^{j}$ are expansion coefficients of the $\mathrm{H}_{2} \mathrm{O}$ wave functions in the II $R$ representation over the symmetric top wave functions $|j k m\rangle$ with $k=-j,-j+1, \cdots$, $j$ such that

$\left|j \tau m>=\sum_{k} U_{k \tau}^{j}\right| j k m>$. 


\subsection{Fourier transforms of the correlations and subsequent Hilbert transforms}

As further steps in developing $S_{2 o u t e r, i}\left(r_{c}\right)$ from Eq. (11), we apply two tools, i.e., the Fourier transform and the Hilbert transform. First of all, by introducing $H_{L_{1} K_{1} K_{1}^{\prime} L_{2}}(\omega)$, the Fourier transforms of the correlation functions, defined by

$H_{L_{1} K_{1} K_{1}^{\prime} L_{2}}(\omega)=\frac{1}{\sqrt{2 \pi}} \int_{-\infty}^{\infty} e^{i \omega t} F_{L_{1} K_{1} K_{1}^{\prime} L_{2}}(t) d t$,

one is able to obtain an expression for the real part of $S_{2, \text { outer }, i}\left(r_{c}\right)$ as

$$
\begin{aligned}
\operatorname{ReS}_{2, \text { outer }, i}\left(r_{c}\right)= & \sqrt{\frac{\pi}{2}} \sum_{L_{1} K_{1} K_{1}^{\prime} L_{2}} \sum_{j_{i}^{\prime} \tau_{i}^{\prime}}\left(2 j_{i}^{\prime}+1\right) \\
& \times D\left(j_{i} \tau_{i} j_{i}^{\prime} \tau_{i}^{\prime} ; L_{1} K_{1}\right) D\left(j_{i} \tau_{i} j_{i}^{\prime} \tau_{i}^{\prime} ; L_{1} K_{1}^{\prime}\right) \\
& \times \sum_{j_{2} j_{2}^{\prime}}\left(2 j_{2}+1\right)\left(2 j_{2}^{\prime}+1\right) \rho_{j_{2}} C^{2}\left(j_{2} j_{2}^{\prime} L_{2}, 000\right) \\
& \times H_{L_{1} K_{1} K_{1}^{\prime} L_{2}}\left(\omega_{j_{i} \tau_{i} j_{i}^{\prime} \tau_{i}^{\prime}}+\omega_{j_{2} j_{2}^{\prime}}\right) .
\end{aligned}
$$

Secondly, by introducing $I_{L_{1} K_{1} K_{1}^{\prime} L_{2}}(\omega)$, the Hilbert transforms of $H_{L_{1} K_{1} K_{1}^{\prime} L_{2}}(\omega)$, defined by

$I_{L_{1} K_{1} K_{1}^{\prime} L_{2}}(\omega)=\frac{1}{\pi} P \int_{-\infty}^{+\infty} d \omega^{\prime} \frac{1}{\omega-\omega^{\prime}} H_{L_{1} K_{1} K_{1}^{\prime} L_{2}}\left(\omega^{\prime}\right)$,

where $P$ means the principal part, one is able to obtain an expression for $\operatorname{Im} S_{2, \text { outer, } i}\left(r_{c}\right)$ which is the same as $\operatorname{Re} S_{2, \text { ou- }}$ ter, $i\left(r_{c}\right)$ except for a replacement of $H_{L_{1} K_{1} K_{1}^{\prime} L_{2}}\left(\omega_{j_{i} \tau_{j}^{\prime} j_{i}^{\prime} \tau_{i}^{\prime}}+\omega_{j_{2} j_{2}^{\prime}}\right)$ by $I_{L_{1} K_{1} K_{1}^{\prime} L_{2}}\left(\omega_{j_{i} \tau_{j}^{\prime} j_{i}^{\prime}}+\omega_{j_{2} j_{2}^{\prime}}\right)$ in Eq. (17). With respect to expressions for $\operatorname{Re}_{2, \text { outer f } f}\left(r_{c}\right)$ and $\operatorname{Im} S_{2, \text { outer, } f}\left(r_{c}\right)$, they are same as their corresponding partners except for the replacement of the initial quantum numbers $j_{i} \tau_{i,} j^{\prime}{ }_{i}$, and $\tau_{i}^{\prime}$ by the final ones $j_{f} \tau_{f}, j_{f}^{\prime}$, and $\tau_{f}^{\prime}$. Meanwhile, the expression for $S_{2, \text { middle }}\left(r_{c}\right)$, which is real, is given by

$$
\begin{aligned}
S_{2, \text { middle }}\left(r_{c}\right)= & \sqrt{2 \pi} \sum_{L_{1} K_{1} K_{1}^{\prime} L_{2}}\left\{(-1)^{L_{1}}\left(2 j_{i}+1\right)\left(2 j_{f}+1\right)\right. \\
& \left.\times W\left(j_{i} j_{f} j_{i} j_{f} ; 1 L_{1}\right) D\left(j_{i} \tau_{i} j_{i} \tau_{i} ; L_{1} K_{1}\right) D\left(j_{f} \tau_{f} j_{f} \tau_{f} ; L_{1} K_{1}^{\prime}\right)\right\} \\
& \times \sum_{j_{2} j_{2}^{\prime}}\left(2 j_{2}+1\right)\left(2 j_{2}^{\prime}+1\right) \rho_{j_{2}} C^{2}\left(j_{2} j_{2}^{\prime} L_{2}, 000\right) \\
& \times H_{L_{1} K_{1} K_{1}^{\prime} L_{2}}\left(\omega_{j_{2} j_{2}^{\prime}}\right) .
\end{aligned}
$$

After all these expressions are available, we have obtained two basic formulas,

$\operatorname{Re}_{2}\left(r_{c}\right)=\operatorname{Re} S_{2, \text { outer }, i}\left(r_{c}\right)+\operatorname{Re}_{2, \text { outer }, f}\left(r_{c}\right)+S_{2, \text { middle }}\left(r_{c}\right)$,

and

$\operatorname{Im} S_{2}\left(r_{c}\right)=-\operatorname{Im} S_{2, \text { outer }, i}\left(r_{c}\right)+\operatorname{Im} S_{2, \text { outer }, f}\left(r_{c}\right)$,

where the minus sign of $\operatorname{Im} S_{2, \text { outer, } i}\left(r_{c}\right)$ results from the fact that $S_{2}\left(r_{c}\right)=S_{2, \text { outer }, i}\left(r_{c}\right)^{*}+S_{2, \text { outer } f}\left(r_{c}\right)+S_{2, \text { middle }}\left(r_{c}\right)$ [10].

It is worth mentioning that the relationship among these functions is that $H_{L_{1} K_{1} K_{1}^{\prime} L_{2}}(\omega)$ are the Fourier transforms of $F_{L_{1} K_{1} K_{1}^{\prime} L_{2}}(t)$, meanwhile $I_{L_{1} K_{1} K_{1}^{\prime} L_{2}}(\omega)$ are the Hilbert transforms of $H_{L_{1} K_{1} K_{1}^{\prime} L_{2}}(\omega)$. In addition, because $F_{L_{1} K_{1} K_{1}^{\prime} L_{2}}(t)$ are even functions, their Fourier transforms $H_{L_{1} K_{1} K_{1}^{\prime} L_{2}}(\omega)$ are real and even, and the subsequent Hilbert transforms $I_{L_{1} K_{1} K_{1}^{\prime} L_{2}}(\omega)$ are real and odd. Finally, we note that all these functions $F_{L_{1} K_{1} K_{1}^{\prime} L_{2}}(t), H_{L_{1} K_{1} K_{1}^{\prime} L_{2}}(\omega)$, and $I_{L_{1} K_{1} K_{1}^{\prime} L_{2}}(\omega)$ are associated with specified trajectories. As a result, all of them depend on $r_{c}$. For simplicity, we have omitted the argument $r_{c}$ in their notations.

In practice, however, this two steps method may not be the best way to follow. For the first step, usually the continuous Fourier transforms are replaced by the discrete Fourier transforms with proper samplings. The latter can be easily derived with the FFT algorithm [9] which is cited as one of the most significant contributions to numerical analysis of the 20-th century [8]. As a result, there is no obstacle in carrying out this step. However, the second step requires one to evaluate the Cauchy principal integration. Although subroutines for carrying out the Cauchy principal integration are available in main computer libraries, their performances are not always satisfactory. In general, these subroutines work well for most of cases. However, their unstable performances do happen occasionally. There are a lot of independent Hilbert transforms $I_{L_{1} K_{1} K_{1}^{\prime} L_{2}}(\omega)$ and if one considers 132 correlations this number is 39 . In addition, all these functions have two dimensions. In order to depict each of them well, one has to use high resolutions by selecting several hundred points for each of its two variables such that the corresponding range is well covered. As a result, in practical calculations sometimes one needs to evaluate a huge number of the Cauchy principal integrations in the order of $10^{7}$. An obstacle here does not result from the computational burdens, but from unstable performances of the subroutines occasionally happening because one is not able to pick up all unstable results from such huge data pool and to make modifications accordingly. As a result, the ability to derive reliable two dimensional profiles of $I_{L_{1} K_{1} K_{1}^{\prime} L_{2}}(\omega)$ becomes a big challenge. In fact, in spite of that the formalism in calculating the half-widths and the lineshifts based on the coordinate representation has been developed several years ago, [6] we have not reported any calculated results involving evaluations of $I_{L_{1} K_{1} K_{1}^{\prime} L_{2}}(\omega)$. We have presented calculated half-widths for the $\mathrm{H}_{2} \mathrm{O}-\mathrm{N}_{2}$ system [7], but we have assumed there that contributions from $\operatorname{ImS}_{2}\left(r_{c}\right)$ to calculated half-widths can be ignored. Meanwhile, we have not reported any calculated shifts at all. In summary, in order to make progress, to find an alternative way to evaluate $I_{L_{1} K_{1} K_{1}^{\prime} L_{2}}(\omega)$ becomes mandatory. Recently, we began to wonder whether taking the two steps is the only way to find $I_{L_{1} K_{1} K_{1}^{\prime} L_{2}}(\omega)$ or can one derive them directly from $F_{L_{1} K_{1} K_{1}^{\prime} L_{2}}(t)$.

\section{Causal correlation functions and Fourier transforms}

\subsection{Causal correlation and Fourier transform}

We briefly explain a concept about the causal function and related topics which have been widely used in signal processing and many other engineering and science disciplines. First of all, let us assume $F(t)$ of interest is an even function. We want to find its Fourier transform $H(\omega)$ together with the subsequent Hilbert transform $I(\omega)$. The usual route which is depicted in Fig. 1 is to carry out the Fourier transform of $F(t)$ first, then one performs a subsequent Hilbert transform of $H(\omega)$ to derive $I(\omega)$. A question arises that is there a way to establish a direct link between $F(t)$ and $I(\omega)$ ? 


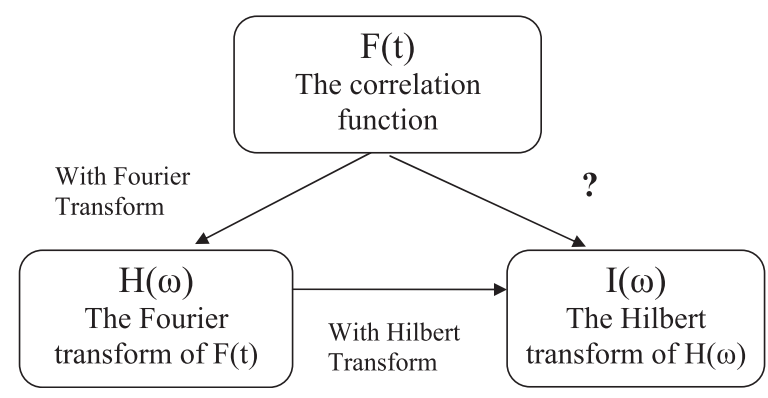

Fig. 1. A diagram to show the usual route to derive the Fourier transform $H(\omega)$ from the function $F(t)$ and a subsequent Hilbert transform $I(\omega)$ of $H(\omega)$. Is there a way to establish a direct link between $F(t)$ and $I(\omega)$ ?

Because the Hilbert transform including the KramersKronig relations associated with it has been applied in solving many engineering and science problems, we believe that by searching tools used in other disciplines, one may be able to find a useful one. Fortunately, the useful tool does exist in other fields such as signal processing [17]. By adopting this method, instead of starting from the function $F(t)$ itself one defines its causal function denoted by $\tilde{F}(t)$ as

$\tilde{F}(t)=F(t) \theta(t)$,

where $\theta(t)$ is the unit step function (the Heaviside step function) defined by

$\theta(t)=1 \quad t \geq 0$,

$\theta(t)=0 \quad t<0$.

Then, one considers the Fourier transform of the causal function $\tilde{F}(t)$ which can be expressed by a convolution of the Fourier transforms of its products, i.e., $H(\omega)$ and $\Theta(\omega)$,

$\tilde{H}(\omega)=\frac{1}{\sqrt{2 \pi}} \int_{-\infty}^{\infty} \tilde{F}(t) e^{i \omega t} d t=\frac{1}{\sqrt{2 \pi}} \int_{-\infty}^{\infty} H\left(\omega^{\prime}\right) \Theta\left(\omega-\omega^{\prime}\right) d \omega^{\prime}$.

It is worth mentioning that because $\tilde{F}(t)$ is neither even nor odd, $\tilde{H}(\omega)$ is a complex function. On the other hand, the Fourier transform of $\theta(t)$ is well known and can be expresses as

$\Theta(\omega)=\frac{1}{\sqrt{2 \pi}}\left(\pi \delta(\omega)+i P \frac{1}{\omega}\right)$,

where $P$ means to take the Cauchy principal value upon integration. With Eq. (25), one can rewrite Eq. (24) as

$$
\begin{aligned}
\tilde{H}(\omega) & =\frac{1}{2 \pi} \int_{-\infty}^{\infty} H\left(\omega^{\prime}\right)\left[\pi \delta\left(\omega-\omega^{\prime}\right)+i P \frac{1}{\omega-\omega^{\prime}}\right] d \omega^{\prime} \\
& =\frac{1}{2}\left[H(\omega)+i \frac{1}{\pi} P \int_{-\infty}^{\infty} \frac{H\left(\omega^{\prime}\right)}{\omega-\omega^{\prime}} d \omega^{\prime}\right] .
\end{aligned}
$$

With Eq. (26), one can conclude that $H(\omega)$ and $I(\omega)$, the Fourier transform of the function $F(t)$ and the subsequent Hilbert transform, equal to twice the real and imaginary parts of the Fourier transform of the causal function $\tilde{F}(t)$,

$H(\omega)=2 \operatorname{Re} \tilde{H}(\omega)$,

$I(\omega)=2 \operatorname{Im} \tilde{H}(\omega)$.

As a result, by just taking one step, one is able to derive $H(\omega)$ and $I(\omega)$ simultaneously. In comparison with the usual method, the new method enables one to reduce two steps to one. But the most important advantage of the new method is its ability to evaluate Cauchy principal integrations without carrying out these integrations themselves at all. As mentioned above, thanks for the powerful FFT, the remaining step can be well accomplished. In fact, the computer codes used here are the same as those used in completing the first step of the previous method.

Of course, the success in applying the new method here depends on how accurately the Hilbert transform $I(\omega)$ can be obtained. Before we present test results to demonstrate accuracies of this method could achieve, we briefly explain a technique used to derive the Fourier transform of a function $F(t)$. This technique has been successfully used to derive $H(\omega)$ in our previous half-width calculations [6,7] and will be used in calculating $\tilde{H}(\omega)$ here.

\subsection{Sampling the correlations and calculating the Fourier transforms}

The method used to derive the Fourier transform of a function is based on the sampling theory. Readers can find detailed explanations about this technique in the book by Weaver [8]. The basic idea is by sampling, one converts a function of interest to a sequence, calculates its discrete Fourier transform, and then relates this transformed sequence to the Fourier transform of the original function.

When one samples a function $F(t)$ with a sampling rate $\Delta t$, one converts $F(t)$ to a sequence $\{F(n)\}$ whose terms are values of $F(t)$ at the discrete locations $t=n \Delta t$. According to the Whittaker-Shannon sampling theorem, if the function $F(t)$ is band limited with handwidth $\Omega_{f}$ and the sampling rate is chosen such that $\Delta t=1 /\left(2 \Omega_{f}\right)$ called as the Nyquist rate, then $F(t)$ can be recovered uniquely and exactly from the sampled sequence $\{F(n)\}$. We note that a function is called "band limited with bandwidth $\Omega_{f}$ " if its Fourier transform $H(\omega)$ has bounded support over the interval $\left[-\Omega_{f}, \Omega_{f}\right]$.

Usually both the frequency f and the angular frequency $\omega$ are referred by people as the frequency. In order to avoid confusion, we use the notation $\Omega_{f}$ to indicate the one associated with the frequency f, but not the angular frequency $\omega$. However, because many spectroscopists prefer to use the angular frequency $\omega$ in their studies, we follow their preferences here. Then, the interval should be understood as $\left[-2 \pi \Omega_{f}, 2 \pi \Omega_{f}\right]$.

One assumes the function $F(t)$ has bounded support over the time domain $[-T, T]$ and as well as being band limited over $\left[-2 \pi \Omega_{f}, 2 \pi \Omega_{f}\right]$. Because the correlation functions introduced in calculating the half-widths and lineshifts are smooth functions and their magnitudes decrease very quickly to zero as $t$ increases, we believe this assumption is applicable for all of them. First, one samples $F(t)$ with the Nyquist rate $\Delta t=1 /\left(2 \Omega_{f}\right)$ and chooses the number of samples such that $N \Delta t \geq 2 T$ and obtains a sequence $\{F(n)\}$. According to the sampling theory, the original function $F(t)$ can be completely recovered from $\{F(n)\}$ such that

$F(t)=\sum_{n=0}^{N-1} F(n) \sin c\left(2 \pi \Omega_{f}(t-n \Delta t)\right)$. 
Then, by taking the Fourier transform both side of the above equation, one can obtain

$H(\omega)=\frac{1}{2 \Omega_{f}} \sum_{n=0}^{N-1} F(n) e^{-i \omega n \Delta t}$.

Next, one samples $H(\omega)$ with $\Delta \omega=2 \pi /(2 T)$ (i.e., with the Nyquist rate $\left.\Delta f=1 /(2 T)=2 \Omega_{f} / N\right)$ and obtains another sequence in the frequency domain

$$
\begin{aligned}
H(m) & =\frac{1}{2 \Omega_{f}} \sum_{n=0}^{N-1} F(n) e^{-i m n \Delta \omega \Delta t}=N \Delta t \cdot\left\{\frac{1}{N} \sum_{n-0}^{N-1} F(n) e^{-2 \pi i m n / N}\right\} \\
& =N \Delta t \cdot \mathcal{F}\{F(n)\},
\end{aligned}
$$

where $\mathcal{F}\{F(n)\}$ denotes the discrete Fourier transform of $\{F(n)\}$. With Eq. (30), one can conclude that the sequence $\{H(m)\}$ are equal to the discrete Fourier transform of the sequence $\{F(n)\}$ multiplied by $N \Delta t$. Meanwhile, because one chooses the Nyquist rate in deriving the sampling sequence $\{H(m)\}$, it is guaranteed by the sampling theorem that the Fourier transform $H(\omega)$ can be derived from $\{H(m)\}$ without any distortions. Now, we have completed a brief explanation about how to effectively and accurately derive the Fourier transform for the function $F(t)$.

In our formalism to calculate the half-widths and lineshifts, instead of $t$ and $\omega$ we prefer to use the dimensionless arguments $z$ and $k$ as the argument of the correlation functions, and the argument of their Fourier transforms and the subsequent Hilbert transforms. According to our numerical tests, magnitudes of the correlation functions could decrease by about 5-7 orders as $z$ changes from 0 to 12.8. In fact, when $\mathrm{z}$ increases from 0 to 12.8 , the distance between two interacting molecules have increased from the closest distance $r_{c}$ to $12.8 \times r_{c}$. Roughly speaking, the latter is at least about $40 \AA$ and at this distance interaction between the two molecules is almost negligible. Therefore, we can consider the correlation functions are limited in the range $[-Z, Z]=[-12.8,12.8]$. In practice, it is better to use finer sampling rates $\Delta z$ and larger numbers $N$ such that $N \Delta z>2 Z$. In the Section 3.3, we will present several samples to show how this method works. Finally, it is worth mentioning that the range of $k$ accessible from this method is $[-\pi / \Delta z, \pi / \Delta z]$ and one has to make sure that the $\Delta z$ is small enough that this range would be larger than the whole $k$ range of interest.

\subsection{The accuracy check of calculated Hilbert transform}

As the first example, we consider the function $F(t)$ to be a Gaussian

$F(t)=e^{-\left(t^{2} / 2\right)}$,

because its Fourier transform $H(\omega)$ is also a Gaussian

$H(\omega)=e^{-\left(\omega^{2} / 2\right)}$,

and the subsequent Hilbert transform $I(\omega)$ is well known. In fact, the Hilbert transform of a Gaussian is a function called as the Dawson's integral and it can also be related to the imaginary error function. In applying the new method, one can introduce the corresponding causal function

$\tilde{F}(t)=e^{-\left(t^{2} / 2\right)} \theta(t)$.

Then, after carrying out the Fourier transform of $\tilde{F}(t)$ with the technique described above, the Hilbert transform of $H(\omega)$ can be obtained from the imaginary part of this Fourier transform. Because values of the Dawson's integral are known, by comparing calculated values from $\tilde{F}(t)$ with them, one can check how accurate the calculated values are.

By setting $T=12.8$ and $N \Delta t=8 \mathrm{~T}$, we have calculated the Fourier transform of $\tilde{F}(t)$ and obtained $I(\omega)$ in the $\omega$ range of $[0,1000]$ with several different choices of $N$ and we list some of the results in Table 1 together with the

Table 1

\begin{tabular}{|c|c|c|c|c|}
\hline \multirow[t]{2}{*}{$\omega$} & \multirow[t]{2}{*}{ Dawson's integral } & \multicolumn{3}{|l|}{ Calculated $I(\omega)$} \\
\hline & & $\begin{array}{l}N=2097152 \\
\Delta t=0.1 \times 2^{-11}\end{array}$ & $\begin{array}{l}N=131072 \\
\Delta t=0.1 \times 2^{-7}\end{array}$ & $\begin{array}{l}N=65536 \\
\Delta t=0.1 \times 2^{-6}\end{array}$ \\
\hline 1.0 & $0.578290 \mathrm{E}+00$ & $0.578290 \mathrm{E}+00$ & $0.578290 \mathrm{E}+00$ & $0.578290 \mathrm{E}+00$ \\
\hline 10.0 & $0.806116 \mathrm{E}-01$ & $0.806116 \mathrm{E}-01$ & $0.806118 \mathrm{E}-01$ & $0.806124 \mathrm{E}-01$ \\
\hline 20.0 & $0.399947 E-01$ & $0.399947 \mathrm{E}-01$ & $0.399951 \mathrm{E}-01$ & $0.399963 E-01$ \\
\hline 30.0 & $0.266258 \mathrm{E}-01$ & $0.266258 \mathrm{E}-01$ & $0.266264 \mathrm{E}-01$ & $0.266282 \mathrm{E}-01$ \\
\hline 40.0 & $0.199596 \mathrm{E}-01$ & $0.199596 \mathrm{E}-01$ & $0.199604 \mathrm{E}-01$ & $0.199628 \mathrm{E}-01$ \\
\hline 50.0 & $0.159641 \mathrm{E}-01$ & $0.159640 \mathrm{E}-01$ & $0.159651 \mathrm{E}-01$ & $0.159681 \mathrm{E}-01$ \\
\hline 60.0 & $0.133018 E-01$ & $0.133017 \mathrm{E}-01$ & $0.133029 E-01$ & $0.133066 \mathrm{E}-01$ \\
\hline 70.0 & $0.114007 \mathrm{E}-01$ & $0.114006 \mathrm{E}-01$ & $0.114020 \mathrm{E}-01$ & $0.114063 E-01$ \\
\hline 80.0 & $0.997512 E-02$ & $0.997502 \mathrm{E}-02$ & $0.997664 \mathrm{E}-02$ & $0.998152 \mathrm{E}-01$ \\
\hline 90.0 & $0.886648 E-02$ & $0.886639 \mathrm{E}-02$ & $0.886821 \mathrm{E}-02$ & $0.887369 \mathrm{E}-02$ \\
\hline 100.0 & $0.797964 \mathrm{E}-02$ & $0.797954 \mathrm{E}-02$ & $0.798156 \mathrm{E}-02$ & $0.798765 E-02$ \\
\hline 200.0 & $0.398952 E-02$ & $0.398940 \mathrm{E}-02$ & $0.399344 \mathrm{E}-02$ & $0.400566 \mathrm{E}-02$ \\
\hline 300.0 & $0.265964 \mathrm{E}-02$ & $0.265964 \mathrm{E}-02$ & $0.266571 \mathrm{E}-02$ & $0.268412 \mathrm{E}-02$ \\
\hline 400.0 & $0.199472 E-02$ & $0.199475 E-02$ & $0.200285 E-02$ & $0.202755 E-02$ \\
\hline 500.0 & $0.159578 E-02$ & $0.159581 \mathrm{E}-02$ & $0.160596 \mathrm{E}-02$ & $0.163708 \mathrm{E}-02$ \\
\hline 600.0 & $0.132981 \mathrm{E}-02$ & $0.132985 E-02$ & $0.134206 \mathrm{E}-02$ & $0.137978 \mathrm{E}-02$ \\
\hline 700.0 & $0.113984 \mathrm{E}-02$ & $0.113989 E-02$ & $0.115416 \mathrm{E}-02$ & $0.119870 \mathrm{E}-02$ \\
\hline 800.0 & $0.997357 E-03$ & $0.997419 \mathrm{E}-03$ & $0.101378 E-02$ & $0.106537 \mathrm{E}-02$ \\
\hline 900.0 & $0.886539 E-03$ & $0.886610 \mathrm{E}-03$ & $0.905067 \mathrm{E}-03$ & $0.964032 E-03$ \\
\hline 1000.0 & $0.797885 E-03$ & $0.797964 \mathrm{E}-03$ & $0.818543 \mathrm{E}-03$ & $0.885226 \mathrm{E}-03$ \\
\hline
\end{tabular}

Values of $I(\omega)$ derived from using different sampling number $N$. 
values of the Dawson's integral. As shown in the table, the method works excellently. In general, the smaller $\omega$ is, the higher the accuracy of $I(\omega)$. In addition, the larger the $N$ is, the higher the accuracy. For the highest choice of $N=2097152\left(=2^{21}\right)$ and $\Delta t=0.1 \times 2^{-11}$, the error is always less than $0.01 \%$ in the whole $\omega$ range. With the choice of $N=262144\left(=2^{18}\right)$ and $\Delta t=0.1 \times 2^{-8}$ whose results are not listed here, the errors at $\omega=10,100$, and 1000 are $0.0002 \%, 0.005 \%$, and $0.64 \%$, respectively. With the moderate choice of $N=131072 \quad\left(=2^{17}\right)$ and $\Delta \mathrm{t}=0.1 \times 2^{-7}$, the errors at $\omega=10,100$, and 1000 are $0.0002 \%, 0.024 \%$, and $2.59 \%$, respectively. Finally, using the lowest one of $N=65536\left(=2^{16}\right)$ and $\Delta t=0.1 \times 2^{-6}$, these errors become $0.001 \%, 0.1 \%$ and $11 \%$. With respect to $H(\omega)$, our tests show that the calculated results can also match the exact Gaussian function of $\exp \left(-\omega^{2} / 2\right)$ very well. More specifically, as magnitudes of $H(\omega)$ reduce by 5 orders from its maximum (i.e., 1 at $\omega=0$ ), the errors still remain less than $1 \%$.

As a second test, we consider a more realistic problem. There are many early studies of broadened half-widths and induced shifts for two interacting molecules $[16,20]$. In these works, by considering simple potential models consisting of only electric multipole interactions and choosing simple trajectory models, such as the straight line or the parabolic trajectory, people have introduced resonance functions and developed methods to evaluate these functions. Thanks to their contributions, those resonance functions associated with the dipole-dipole, the dipole-quadrupole, and the quadrupole-quadrupole interactions are available in the literature [18-22]. With our formalism, we consider a pair of two linear molecules, choose the parabolic trajectory model and a simple potential model consisting of only the dipole-dipole, the dipole-quadrupole, and the quadrupole-quadrupole interactions. In this case, the correlation functions have simple properties. There is no interweaving between their distance dependence and their internal degree dependence. Besides, the multipole-moments can be simply factored out from their expressions. By exploiting these properties, one is able to introduce simplified correlation functions $F_{11}(z), F_{12}(z)$, and $F_{22}(z)$ which are common for all trajectories and are independent of the multipole moments. Then, with the new method, by introducing the corresponding causal partners $\tilde{F}_{11}(z), \tilde{F}_{12}(z)$, and $\tilde{F}_{22}(z)$, one can find out their Fourier transforms and obtain $H_{11}(k), I_{11}(k), H_{12}(k), I_{12}(k), H_{22}(k)$, and $I_{22}(k)$ accordingly. It turns out that the latter should match the corresponding resonance functions exactly. Therefore, we can use these resonance functions as benchmarks to check how accurate our results are.

Starting from the causal functions of $\tilde{F}_{11}(z), \tilde{F}_{12}(z)$, and $\tilde{F}_{22}(z)$, we have calculated their Fourier transforms with the choice of $\mathrm{N}=262144$ and $\Delta \mathrm{t}=0.1 \times 2^{-8}$ and the latter's real and imaginary parts are our calculated $\mathrm{H}_{11}(\mathrm{k}), \mathrm{I}_{11}(\mathrm{k})$, $\mathrm{H}_{12}(\mathrm{k}), \mathrm{I}_{12}(\mathrm{k}), \mathrm{H}_{22}(\mathrm{k})$, and $\mathrm{I}_{22}(\mathrm{k})$. Based on the fact that $H_{L_{1} L_{2}}(k)$ are even functions of $k$ and $I_{L_{1} L_{2}}(k)$ are odd ones, one only needs to plot half of them along the positive $k$ axis. Meanwhile, because magnitudes of $I_{L_{1} L_{2}}(k)$ decrease more slowly as $k$ increase than magnitudes of $H_{L_{1} L_{2}}(k)$ do, in order to present their profiles more completely one has to plot $I_{L_{1} L_{2}}(k)$ in a larger range of $k$. We present calculated $H_{11}(k), H_{12}(k)$, and $H_{22}(k)$ together with the corresponding resonance functions in Fig. 2(a), (c), and (e). Meanwhile, we present calculated $I_{11}(k), I_{12}(k)$, and $I_{22}(k)$ together with the corresponding resonance functions in Fig. 2(b), (d), and (f) where the $k$ axis is given on a logarithmic scale. As shown in Fig. 2(a)-(f), these $H_{L_{1} L_{2}}(k)$ and $I_{L_{1} L_{2}}(k)$ derived from the causal correlations $\tilde{F}_{L_{1} L_{2}}(z)$ match the corresponding resonance functions very well. Finally, there is a small structure of the resonance function associated with the quadrupole-quadrupole interaction appearing at around $k=7.5$ in Fig. 2(f). This minor structure indicates the formula used to represent this resonance function contains a small error.

\subsection{Samples of $H_{L_{1} K_{1} K_{1}^{\prime} L_{2}}(k)$ and $I_{L_{1} K_{1} K_{1}^{\prime} L_{2}}(k)$ derived from the causal correlations $\tilde{F}_{L_{1} K_{1} K_{1}^{\prime} L_{2}}(z)$}

First of all, we would like to emphasize that when the potential models contain the atom-atom component, the distance and the internal degree dependences of the correlations become interweaving. As a result, the correlations $F_{L_{1} K_{1} K_{1}^{\prime} L_{2}}(z)$ and their associates depend on the parameter $r_{c}$ used in labeling collision trajectories. For simplicity, we have often omitted this argument $r_{c}$ in expressions for these functions, but we will add it in here. Base on the potential model used in updating HITRAN 2008 [14], choosing the 20-th cut-off, including 132 correlations, and selecting the "exact" trajectory model, we have derived functions of $H_{L_{1} K_{1} K_{1}^{\prime} L_{2}}\left(k, r_{c}\right)$ and $I_{L_{1} K_{1} K_{1}^{\prime} L_{2}}\left(k, r_{c}\right)$ from all the causal irreducible correlations $\tilde{F}_{L_{1} K_{1} K_{1}^{\prime} L_{2}}\left(z, r_{c}\right)$ with $L_{1} \leq 4$ and $L_{2} \leq 4$ which are independent. Because magnitudes of $I_{L_{1} K_{1} K_{1}^{\prime} L_{2}}\left(k, r_{c}\right)$ decrease more slowly as $k \rightarrow \infty$ than those of $H_{L_{1} K_{1} K_{1}^{\prime} L_{2}}\left(k, r_{c}\right)$ do, one needs to use larger scales of $k$ in plotting them.

In order to shorten the manuscript, we only present $H_{1002}\left(k, r_{c}\right)$ and $I_{1002}\left(k, r_{c}\right)$ derived from the most important causal correlation $\tilde{F}_{1002}\left(z, r_{c}\right)$ associated with the leading dipole-quadrupole interaction of the $\mathrm{H}_{2} \mathrm{O}$ and $\mathrm{N}_{2}$ pair and other terms with the same symmetry in the spherical expansion of the atom-atom interaction in Figs. 3 and 4. Because $H_{1002}\left(k, r_{c}\right)$ is an even function of $k$ and $I_{1002}\left(k, r_{c}\right)$ is an odd one, one only needs to plot a half part for each of them. More specifically, the range of $k$ used in plotting $I_{1002}\left(k, r_{c}\right)$ in Fig. 4 is $[0,120]$ which is 5 times the range of $k$ (i.e. $[0,24])$ used in plotting $H_{1002}\left(k, r_{c}\right)$ in Fig. 3. With respect to the range of $r_{c}$, because the minimum of the closest distance $r_{c, \min }$ derived from the "exact" trajectory model at $296 \mathrm{~K}$ is $3.5224 \AA$ and the dipole-quadrupole potential component represents a long-range interaction, we have chosen the plotting range of $r_{c}$ is [3.525 $\AA$, $5.025 \AA$ ]. By adopting these range choices, the most important parts of $H_{1002}\left(k, r_{c}\right)$ and $I_{1002}\left(k, r_{c}\right)$ are covered in plotting.

As shown in Figs. 3 and 4, profiles of $H_{1002}\left(k, r_{c}\right)$ and $I_{1002}\left(k, r_{c}\right)$ in the region of $r_{c}>4.0 \AA$ somehow resemble those provided in Fig. 2(c) and (d). This implies that in this region they are mainly determined by the dipolequadrupole interaction and their magnitudes would vary as $r_{c}^{-8}$ as $r_{c} \rightarrow \infty$. On the other hand, in the region of $r_{c}<4.0 \AA$ their profiles contain more structures. 
a

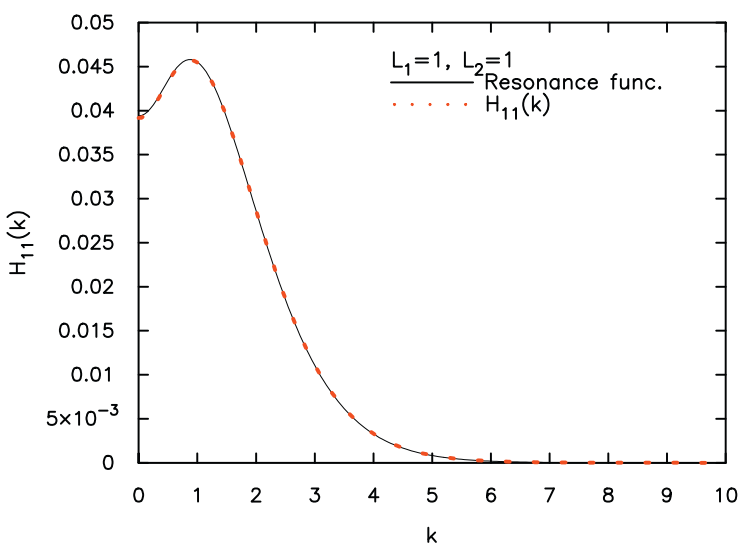

C

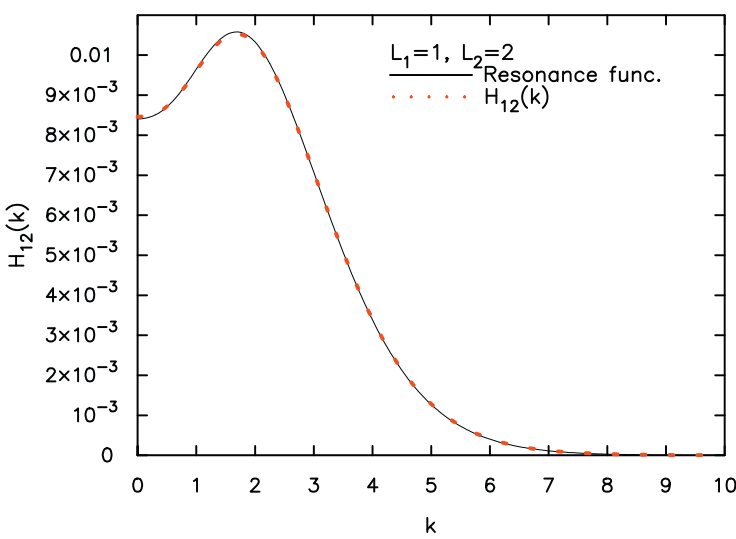

e

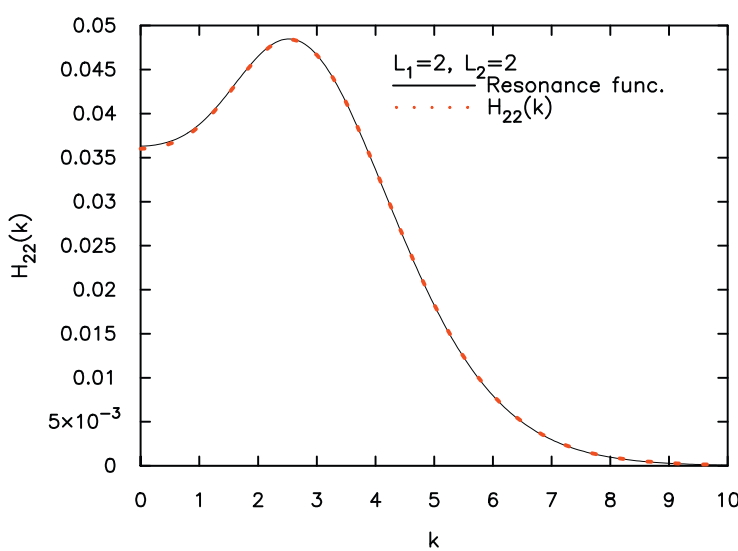

$\mathrm{b}$

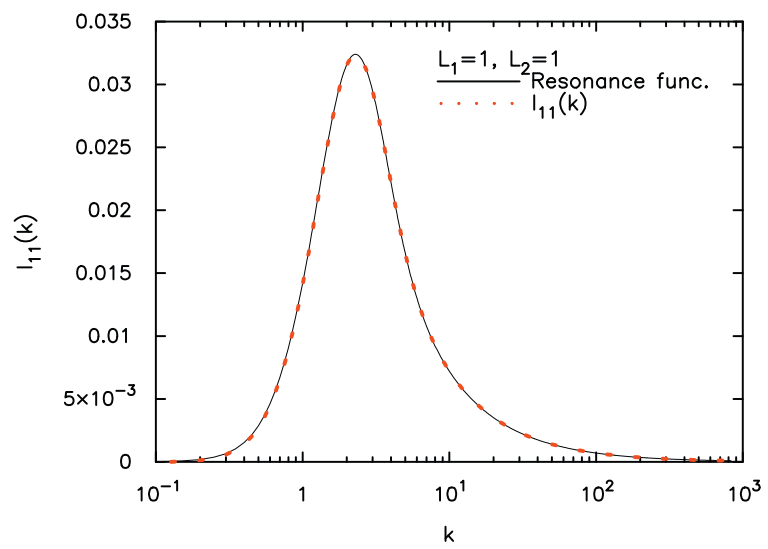

d

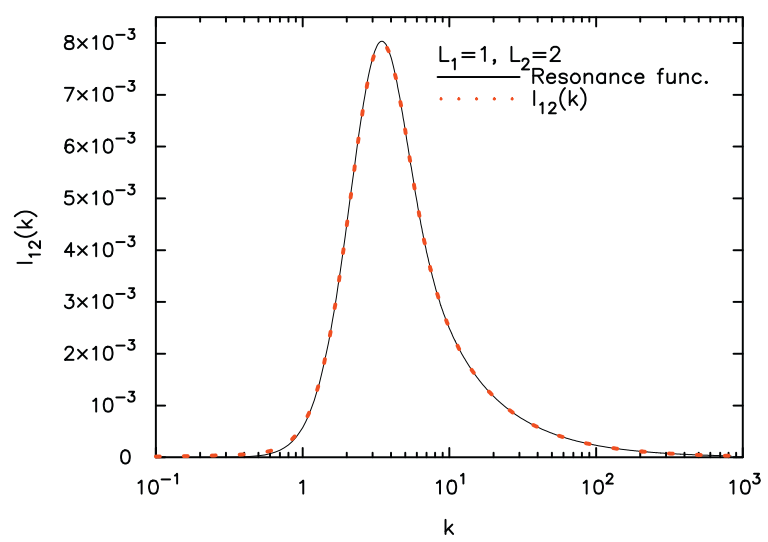

f

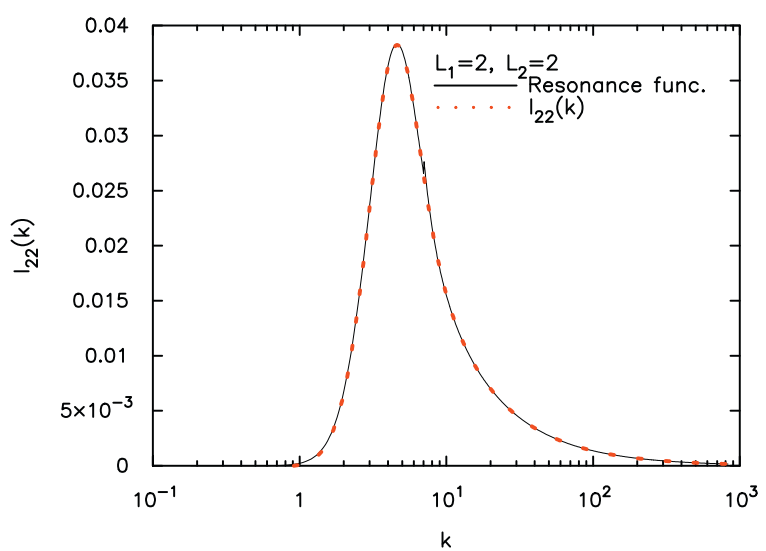

Fig. 2. Calculated $H_{11}(k), I_{11}(k), H_{12}(k), I_{12}(k)$, and $H_{22}(k), I_{22}(k)$ from the causal correlations $\tilde{F}_{11}(z), \tilde{F}_{12}(z)$, and $\tilde{F}_{22}(z)$. They are presented in $($ a)-(f) by red dotted curves. Meanwhile, the corresponding resonance functions available in literary are given by black solid lines.

These structures represent combinations of contributions, not only from the dipole-quadrupole interaction and the related terms in the spherical expansion of the short-range atom-atom interaction, but also couplings between them. In comparison with $H_{1002}\left(k, r_{c}\right)$ shown in Fig. 3, magnitudes of $I_{1002}\left(k, r_{c}\right)$ decrease more slowly such that one have to use a larger scale in plotting Fig. 4.

\section{Calculated $\mathrm{N}_{2}$ induced shifts of $\mathrm{H}_{2} \mathrm{O}$ lines in the pure rotational band}

In the present study, we consider $\mathrm{H}_{2} \mathrm{O}$ lines in the pure rotational band. By making an approximation to replace the integration over the velocity by the averaged velocity $\bar{v}(=\sqrt{8 k T / \pi m})$ and changing the integration variable impact parameter $b$ to the closest distance $r_{c}$ of the 


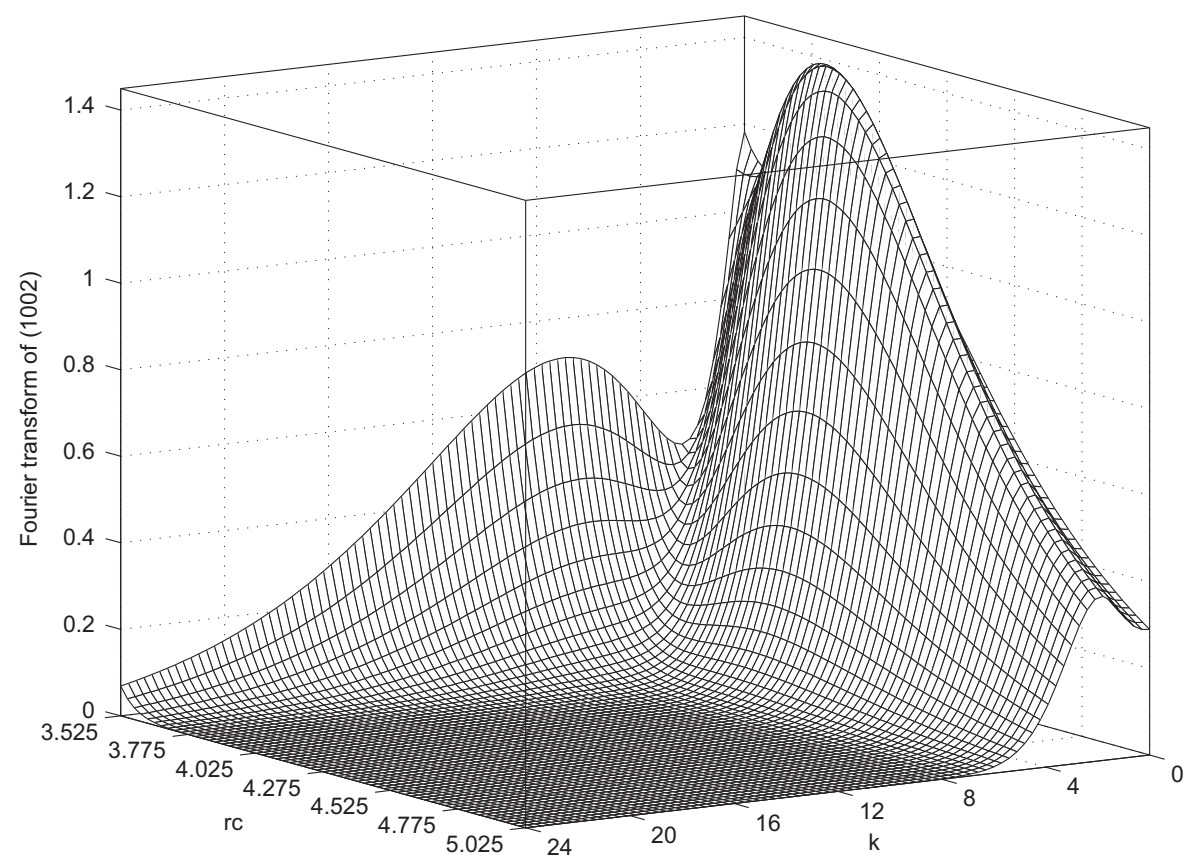

Fig. 3. Fourier transform $H_{1002}\left(k, r_{c}\right)$ (in ps ${ }^{-2}$ ) at $T=296 \mathrm{~K}_{8}$ for a molecular pair of $\mathrm{H}_{2} \mathrm{O}-\mathrm{N}_{2}$ as a two dimensional function of $k$ (dimensionless) and $r_{c}$ (in $\AA$ ). As $r_{c} \rightarrow \infty$, magnitudes of $H_{1002}\left(k, r_{c}\right)$ vary with $r_{c}$ as $r_{c}^{-8}$. This function is derived from the real part of the Fourier transform of the causal correlation $\tilde{F}_{1002}\left(z, r_{c}\right)$ with the choice of $N=262144$ and $\Delta z=0.1 \times 2^{-8}$.

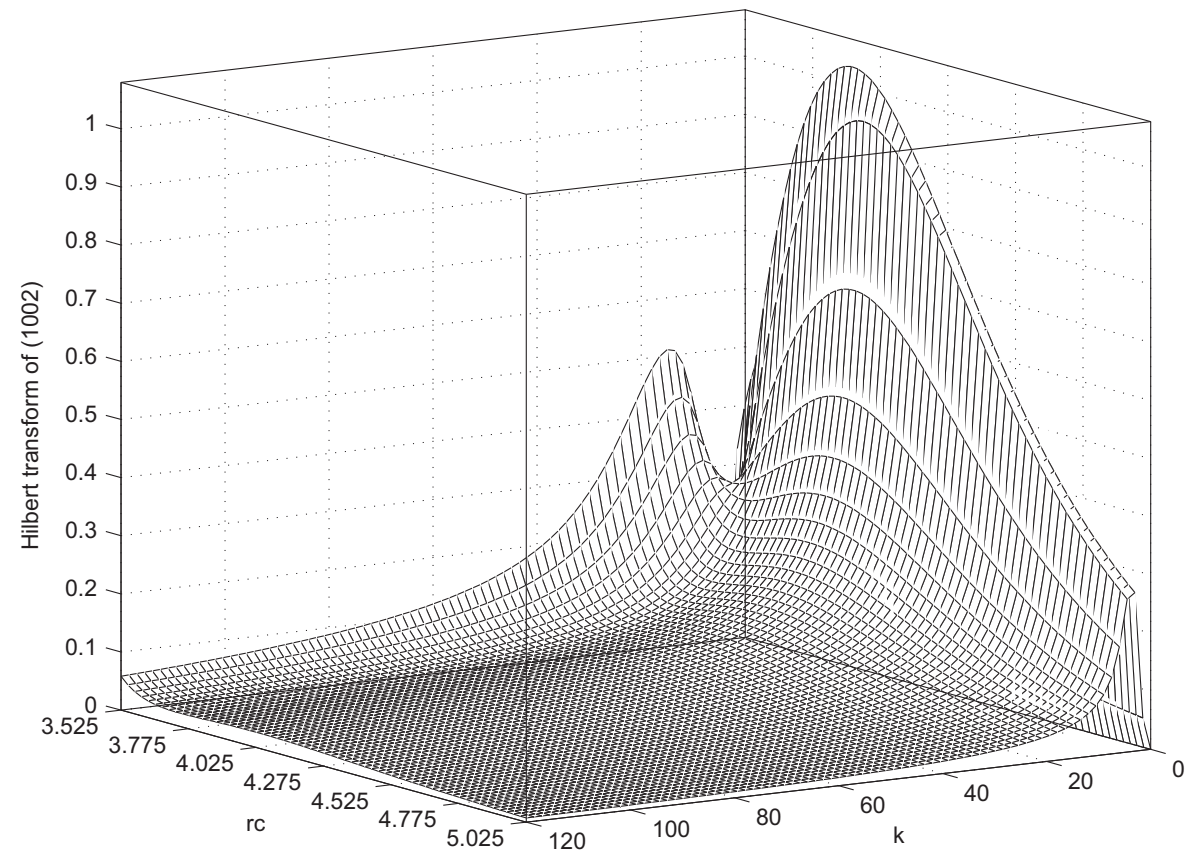

Fig. 4. Same as Fig. 3 except for the Hilbert transform $I_{1002}\left(k, r_{c}\right)$ derived from the imaginary part of the Fourier transform of the causal correlation $\tilde{F}_{1002}\left(z, r_{c}\right)$. As $r_{c} \rightarrow \infty$, magnitudes of $I_{1000}\left(k, r_{c}\right)$ very with $r_{c}$ as $r_{c}^{-8}$.

trajectories, one can obtain simplified expressions for the half-width and the lineshift

$\gamma=\frac{n_{b} \bar{v}}{2 \pi c} \int_{r_{c, \text { min }}}^{+\infty} 2 \pi b\left(\frac{d b}{d r_{c}}\right)\left[1-\cos \left(\operatorname{Im} S_{2}\left(r_{c}\right)\right) e^{-\operatorname{ReS}_{2}\left(r_{c}\right)}\right] d r_{c}$, and

$\delta=\frac{n_{b} \bar{v}}{2 \pi c} \int_{r_{c, \text { min }}}^{+\infty} 2 \pi b\left(\frac{d b}{d r_{c}}\right) \sin \left[\operatorname{Im} S_{2}\left(r_{c}\right)\right] e^{-\mathrm{Re} S_{2}\left(r_{c}\right)} d r_{c}$,

respectively, where $r_{c, \min }$ is the minimum value of $r_{c}$ corresponding to strictly head-on collisions. After $\operatorname{ReS}_{2}\left(r_{c}\right)$ 


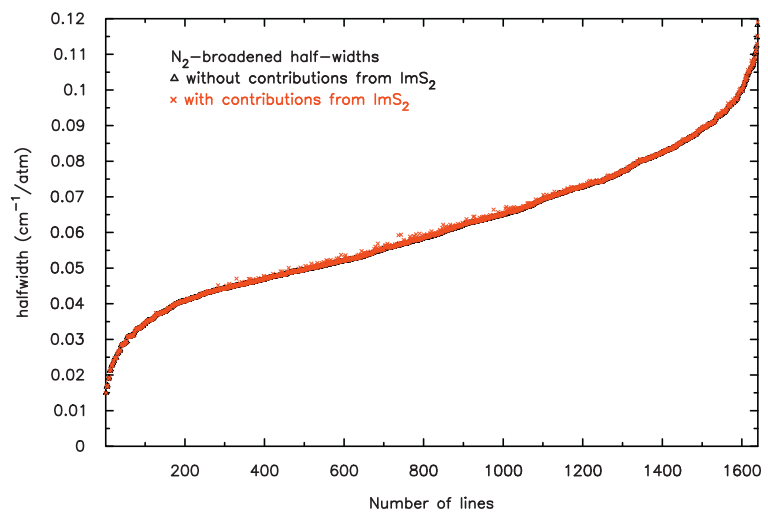

Fig. 5. Comparisons between the calculated $\mathrm{N}_{2}$-broadened half-widths obtained from excluding and including contributions from $\operatorname{Im} S_{2}\left(r_{c}\right)$. They are plotted by $\Delta$ and $\times$, respectively. The 1639 lines in the $\mathrm{H}_{2} \mathrm{O}$ pure rotational band are arranged according to the ascending order of the calculated half-width values without contributions from $\operatorname{Im} S_{2}\left(r_{c}\right)$.

and $\operatorname{Im} S_{2}\left(r_{c}\right)$ of lines of interest are available, one can easily derive their half-widths and shifts from the above two expressions.

\subsection{Contributions from $\mathrm{ImS}_{2}$ (b) to calculated half-widths}

Usually, people assume that effects from the $\operatorname{Im} S_{2}\left(r_{c}\right)$ term can be ignored in calculating the half-width and Eq. (34) can be simplified as [3]

$\gamma=\frac{n_{b} \bar{v}}{2 \pi c} \int_{r_{c, \min }}^{+\infty} 2 \pi b\left(\frac{d b}{d r_{c}}\right)\left[1-e^{-\operatorname{ReS}_{2}\left(r_{c}\right)}\right] d r_{c}$

In order to justify this assumption, we have calculated the $\mathrm{N}_{2}$-broadened half-widths for all $1639 \mathrm{H}_{2} \mathrm{O}$ lines in the pure rotational band listed in HITRAN from both Eqs. (34) and (36) and made comparisons in Fig. 5. Both these two calculations are made based on the potential model used in updating HITRAN 2008 [14] and with the 20-th order cut-off, including 132 correlations, and adopting the parabolic trajectory model. As shown in the figure, the differences between these two results are very small. More specifically, among all the 1639 lines there are only 12 lines with their relative errors above $3 \%$ and the maximum one (happening for the transition of $17_{7,10} \leftarrow$ $17_{4,13}$ at $\left.410.205 \mathrm{~cm}^{-1}\right)$ is $5.1 \%$. Given the fact that the formalisms (i.e., the original and modified RB formalisms) themselves contain other uncertainties larger than the differences reported above and there is an obstacle to accurately derive the $\operatorname{Im} S_{2}\left(r_{c}\right)$ term, to ignore contributions from $\mathrm{ImS}_{2}$ (b) to calculated half-widths is an acceptable and justified approximation. Of course, if one knows how to accurately evaluate $\operatorname{Im} S_{2}\left(r_{c}\right)$ which are necessary for calculations of the lineshifts, it is better to take into account of contributions from $\operatorname{Im} S_{2}\left(r_{c}\right)$ to the half-widths using the Eq. (34).

\subsection{Calculated induced shifts}

Based on the potential model used in updating HITRAN 2008 and with the 20-th order cut-off, including 132 correlations, and adopting the parabolic trajectory model,

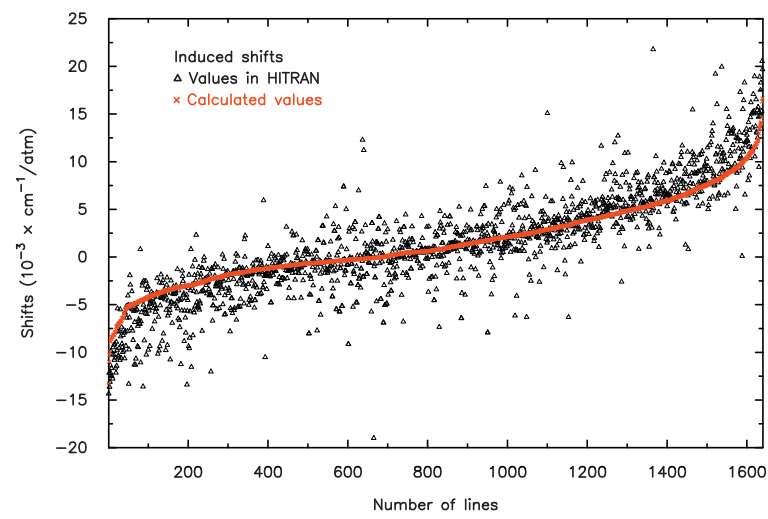

Fig. 6. A comparison between the induced shifts listed in HITRAN 2008 and our calculated values. They are plotted by $\Delta$ and $x$, respectively The 1639 lines in the $\mathrm{H}_{2} \mathrm{O}$ pure rotational band are arranged according to the ascending order of the calculated shift values.

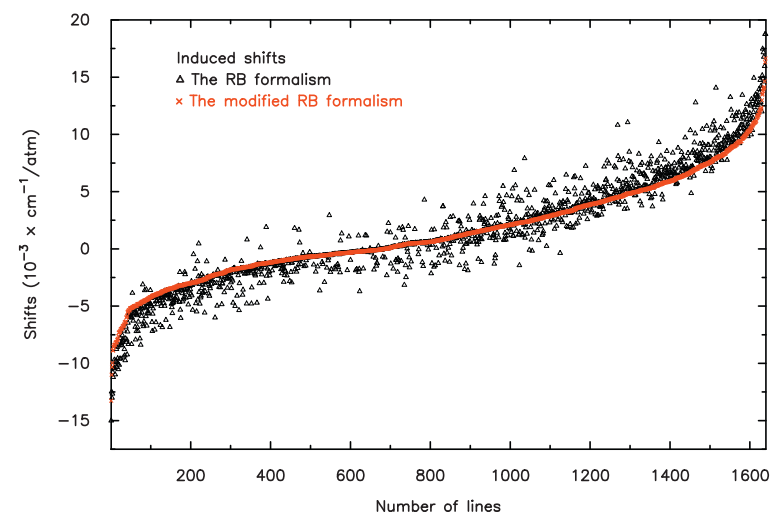

Fig. 7. Comparisons between the calculated $\mathrm{N}_{2}$ induced shifts obtained from the original RB formalism and from the modified version. They are plotted by $\Delta$ and $\times$, respectively. The 1639 lines in the $\mathrm{H}_{2} \mathrm{O}$ pure rotational band are arranged according to the ascending order of the calculated shift values with the modified RB formalism.

we have calculated $\mathrm{N}_{2}$ induced shifts for the $\mathrm{H}_{2} \mathrm{O}$ lines in the pure rotational band. We present our results in Fig. 6 together with those listed in HITRAN 2008. As shown in the figure, there are significant differences between ours and that listed in HITRAN. More specifically, among the 1639 lines there are 649 lines with the relative differences above 50\%, 746 lines with the differences between $10 \%$ and $50 \%$, and only 244 lines with less than $10 \%$. It is worth mentioning that most of the values in HITRAN 2008 come from theoretical calculations [14]. It implies that these two theoretical calculations with the same potential model differ markedly from each other.

We would like to emphasize that in order to make comparisons more meaningful, we assumed the same potential model used in updating HITRAN 2008. Because this potential model is poor [6], we do not think our current calculated shift values are good. Therefore, readers should not consider the predicted results in Fig. 6 as reliable "true" values.

At this stage, we would like to take this opportunity to demonstrate the importance to use the modified RB formalism instead of the original RB one. We present 
comparisons between calculated $\mathrm{N}_{2}$ induced shifts of the $1639 \mathrm{H}_{2} \mathrm{O}$ lines derived from the RB and the modified $\mathrm{RB}$ formalisms in Fig. 7. As shown in the figure, differences between them are pretty large. There are 384 lines with relative errors above 30\%, 767 lines with 5-30\%, and 488 lines with less than $5 \%$. Because both these calculated shift values could be equal to or closer to zero, in order to avoid artificially enlarging error values we define the relative error here by choosing the one with larger magnitude as the denominator. Thus, one can conclude that the effects from the modification on the calculated shifts are significant.

\subsection{Applying the two rules to calculated lineshifts}

Recently, based on the properties of the energy levels and wave functions of $\mathrm{H}_{2} \mathrm{O}$ states, we have established the pair identity and the smooth variation rules applicable for $\mathrm{H}_{2} \mathrm{O}$ lines involving high $\mathrm{j}$ states within individually defined groups such that all their spectroscopic parameters (i.e., the transition wavenumber, intensity, pressure broadened half-width, induced shift, and temperature exponent) must follow them $[23,24]$. We present our calculated shift values of $\mathrm{H}_{2} \mathrm{O}$ lines in three groups of $\left\{j_{0, j^{\prime}} \leftarrow j^{\prime \prime}{ }_{1 j^{\prime \prime}}\right.$, $\left.j^{\prime}{ }_{1 j^{\prime}} \leftarrow j^{\prime \prime}{ }_{0 j^{\prime \prime}}\right\}, \quad\left\{j_{j^{\prime}, 0}^{\prime} \leftarrow j^{\prime \prime} j^{\prime \prime}, 1, \quad j_{j^{\prime}, 1}^{\prime} \leftarrow j^{\prime \prime} j^{\prime \prime}, 0\right\}, \quad$ and $\quad\left\{j^{\prime}{ }_{3 j^{\prime}-2} \leftarrow j^{\prime \prime}{ }_{0 j^{\prime \prime}}\right.$, $\left.j^{\prime}{ }_{2 j^{\prime}-2} \leftarrow j^{\prime \prime}{ }_{1 j^{\prime \prime}}\right\}$ in the $R$ branch, two groups of $\left\{j^{\prime} j^{\prime}, 0 \leftarrow j^{\prime \prime} j^{\prime \prime}-1,1\right.$, $\left.j^{\prime} j^{\prime}, 1 \leftarrow j^{\prime \prime} j^{\prime \prime}-1,2\right\}$ and $\left\{j^{\prime}{ }_{2 j^{\prime}-2} \leftarrow j^{\prime \prime}{ }_{1 j^{\prime \prime}-1}, j^{\prime}{ }_{3 j^{\prime}-2} \leftarrow j^{\prime \prime}{ }_{2 j^{\prime \prime}-1}\right\}$ in the Q branch, and one group of $\left\{j^{\prime}{ }_{2 j^{\prime}}-2 \leftarrow j^{\prime \prime} 1_{j^{\prime \prime}}\right.$ and $\left.j^{\prime}{ }_{3, j^{\prime}-2} \leftarrow j^{\prime \prime}{ }_{0 j^{\prime \prime}}\right\}$ in the P branch in Fig. 8(a)-(f), respectively. Meanwhile, corresponding lineshift values listed in HITRAN 2008 are also presented there. According to our previous studies $[23,24]$, the higher the $j^{\prime \prime}$ is, the better the rules hold. For convenience, one can introduce $j_{b d}$, a low boundary of $j^{\prime \prime}$, for a specified group as a numerical measure that the two rules are applicable for lines in this group whose $j^{\prime \prime}$ values are above the boundary. For the six groups considered here, their suggested boundaries are 7, 3, 13, 5, 13, and 14, respectively $[23,24]$. In Fig. $8(a)-(f)$, we present the calculated shifts of lines whose $j^{\prime \prime}$ values start from these $j_{b d}$ or a little below and up to the highest ones appearing in the list of the pure rotational band in HITRAN 2008.

As shown in Figs. 8(a) and (b) for the first two groups in the $R$ branch, the calculated shifts with $j^{\prime \prime} \geq j_{b d}$ follow the pair identity and the smooth variation rules very well. On the other hand, with respect to those listed in HITRAN 2008 , values of two paired lines with $j^{\prime \prime} \geq j_{b d}$ are almost identical, but they could scatter widely from one pair to its successive pair. This implies that these values follow the first rule, but severely violate the second rule. We note that according to the reference sources in HITRAN 2008 , all of them come from the same source code 37 denoted as recent theoretical calculations [14].

Then, we consider shifts of lines in the third group of the $R$ branch plotted in Fig. 8(c). In general, the calculated shifts follow the two rules well. But, it seems they begin to follow the first rule with a slight delay, starting from $j^{\prime \prime} \geq 14$ instead of the suggested $j^{\prime \prime} \geq 13$. We will explain why this delay could happen later. Concerning values provided in HITRAN 2008, some come from measurements and others from theoretical calculations. In Table 2, we list their reference sources provided by HITRAN 2008.
As shown in Fig. 8(c), except for four pairs of lines with $j^{\prime \prime}=16,18,19$ and 20 , those values with $j^{\prime \prime} \geq j_{b d}$ in HITRAN 2008 violate the pair identity rule. In addition, especially those with $j^{\prime \prime} \geq 17$ do not follow the smooth variation rule as well. Given the fact that usually the lineshifts are very small, of the order of only a few percent of the halfwidths, to measure lineshifts are more difficult than halfwidths. In addition, the lines with high $j^{\prime \prime}$ are very weak, and to perform lineshift measurements becomes a bigger challenge and, therefore, measured values contain large uncertainties. As a result, it is understandable that measured data do not follow the rules well. Besides, mixtures of different sources within the same groups also play a role to make the violations even worse here. It is better to adopt values from the same sources, as much as possible, for the spectroscopic parameters of lines within the same groups in developing databases.

Next, for lines in the first group of the $\mathrm{Q}$ branch plotted in Fig. 8(d), the calculated shifts follow the two rules starting from $j^{\prime \prime} \geq 5$ as expected. In this group, all the values listed in HITRAN 2008 come from the same source [14], i.e., the reference code 37. As shown in the figure, these values follow the pair identity rule, but somehow violate the smooth variation rule. More specifically, it seems that as $j^{\prime \prime} \leq 10$, the values in HITRAN 2008 follow the same trend as ours, but the values with $j^{\prime \prime}>10$ behave strangely.

Finally, for lines in the second group of the $Q$ branch and lines in the group of the $\mathrm{P}$ branch, results are presented in Fig. 8(e) and (f). As shown in these two figures, the calculated shifts follow the two rules. However, they begin to follow the pair identity rule with a delay, i.e., roughly speaking starting from $j^{\prime \prime} \geq 16$ instead of $j^{\prime \prime} \geq 13$ and $j^{\prime \prime} \geq 14$, respectively. Regarding the second rule, there is no such delay appearing here. With respect to the values listed in HITRAN 2008, in general they follow the first rule, but fail to follow the second one. We note that similar to Fig. 8(a), (b), and (d), all these values come from the reference source 37 .

In summary, the presented calculated shifts follow the two rules well. However, passing this test does not mean our calculated values are correct. In fact, unless one has made mistakes in deriving energy levels and wave functions for the $\mathrm{H}_{2} \mathrm{O}$ molecule or made inconsistent errors somewhere else in calculations, calculated results from any self-consistent theories should automatically follow these rules. In contrast, those shift values listed in HITRAN 2008 sometimes fail to follow the two rules, especially the second one. It is worth mentioning that except for several values in the third group of the $R$ branch, all others come from the same theoretical calculations (i.e., the source code 37). As explained above, due to containing large uncertainties, measured data usually do not follow the rules well. However, for theoretically calculated shifts of lines involving high $j$ states, their failure to follow the rules is fatal because usual approximations introduced in calculations such as simplified potential and trajectory models, lower order cut-offs, and so on would not cause the failure. Therefore, one should consider the failure as a clear and strong warming sign of existing inconsistent mistakes in formulas used or bugs in computer codes. 
a

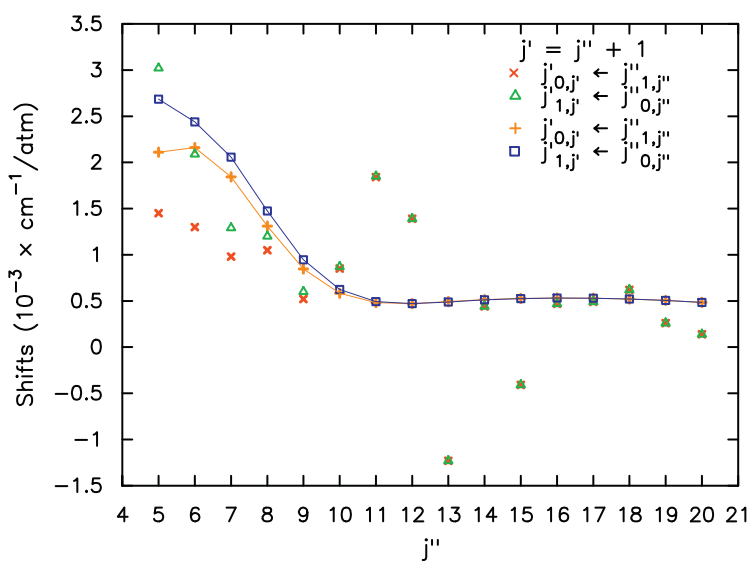

C

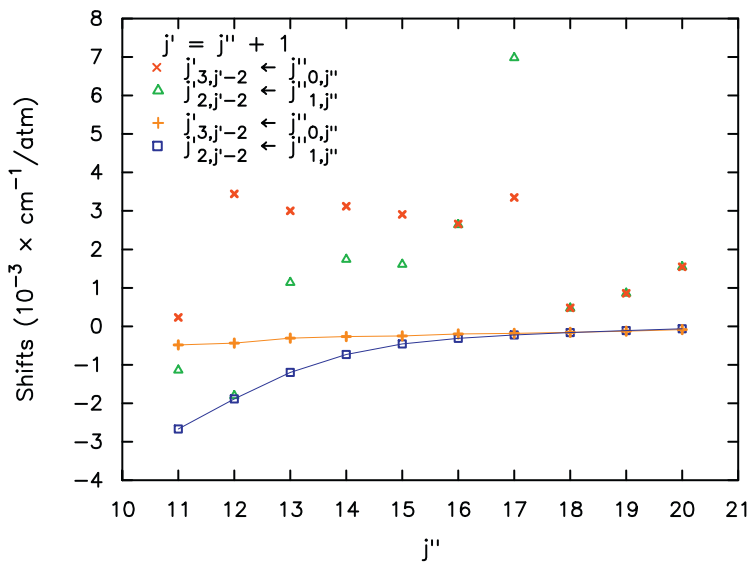

e

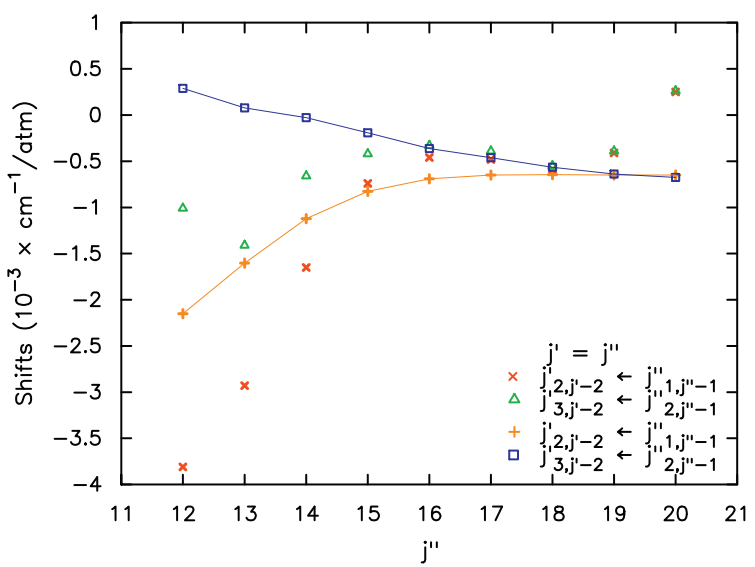

b

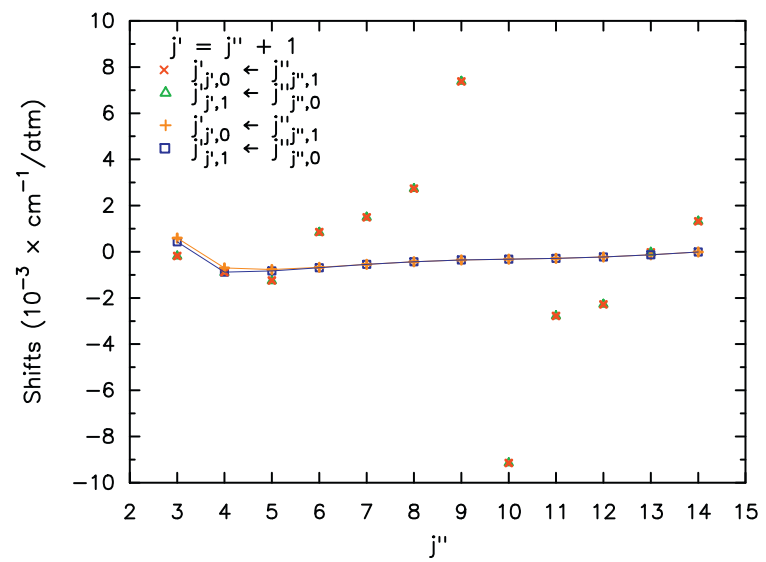

d

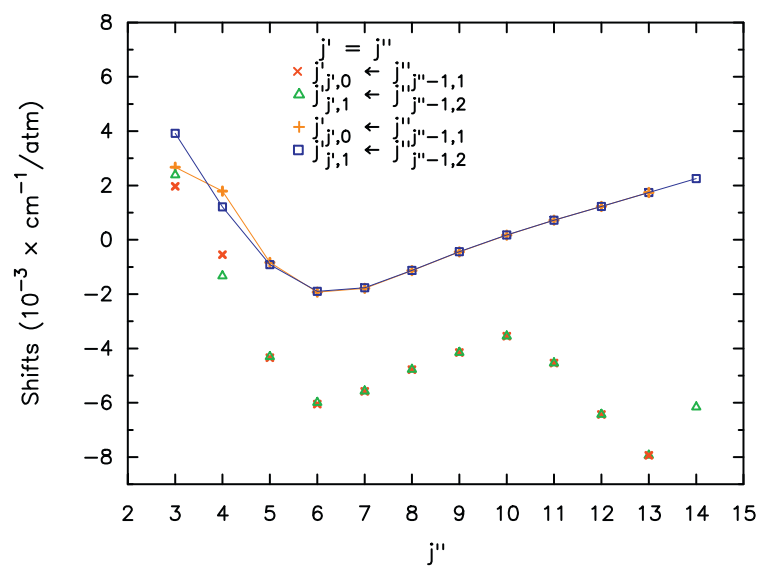

f

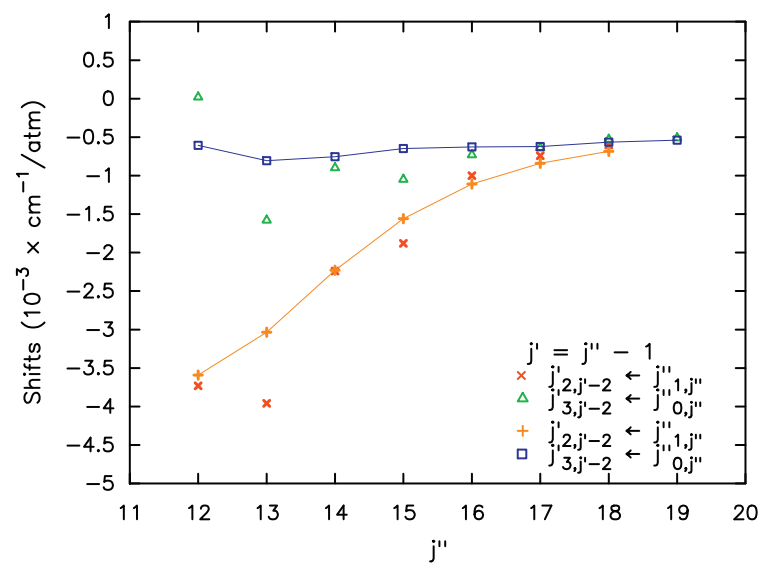

Fig. 8. Induced shifts for three groups of paired lines $\left\{j^{\prime} 0_{j^{\prime}} \leftarrow j^{\prime \prime}{ }_{1 j^{\prime \prime}}, j^{\prime}{ }_{1 j^{\prime}} \leftarrow j^{\prime \prime}{ }_{0, j^{\prime \prime}}\right\},\left\{j_{j^{\prime}, 0}^{\prime} \leftarrow j^{\prime \prime} j^{\prime \prime}, 1\right.$ and $\left.j_{j^{\prime}, 1}^{\prime} \leftarrow j^{\prime \prime} j^{\prime \prime}, 0\right\}$, and $\left\{j^{\prime} j_{j^{\prime}-2} \leftarrow j^{\prime \prime} 0 j^{\prime \prime}\right.$ and $\left.j^{\prime}{ }_{2, j^{\prime}-2} \leftarrow j^{\prime \prime}{ }_{1 j^{\prime \prime}}\right\}$ in the $R$

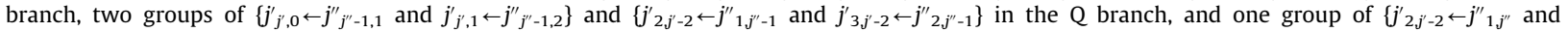
$\left.j^{\prime}{ }_{3 j^{\prime}-2} \leftarrow j^{\prime \prime}{ }_{0, j^{\prime \prime}}\right\}$ in the P branch. They are presented in Fig. 8(a)-(f), respectively. In these plots, the calculated results are given by + and $\square$ and they are connected by two solid color lines. Meanwhile, the values listed in HITRAN 2008 are plotted by $\times$ and $\Delta$.

Before ending this section, we would like to say a few words about the applicability of the rules. In general, based on the black box theory introduced in our previous study [23], one should expect that all the spectroscopic parameters of $\mathrm{H}_{2} \mathrm{O}$ lines involving high $\mathrm{j}$ states follow these two rules. However, how high is high enough to guarantee the applicability of the two rules could differ slightly between the two rules themselves and among the different spectroscopic parameters of interest. As a convenient numerical measure, the so called boundary $j_{b d}$ is 
Table 2

Reference sources of the third group in the $R$ branch.

\begin{tabular}{|c|c|c|c|c|c|}
\hline Lines & Sources* & Lines & Sources* & Lines & Sources* \\
\hline $12_{3,10} \leftarrow 11_{0,11}$ & 37 & $16_{3,14} \leftarrow 15_{0,15}$ & 29 & $20_{3,18} \leftarrow 19_{0,19}$ & 37 \\
\hline $12_{2,10} \leftarrow 11_{1,11}$ & 29 & $16_{2,14} \leftarrow 15_{1,15}$ & 29 & $20_{2,18} \leftarrow 19_{1,19}$ & 37 \\
\hline $13_{3,11} \leftarrow 12_{0,12}$ & 29 & $17_{3,15} \leftarrow 16_{0,16}$ & 6 & $21_{3,19} \leftarrow 20_{0,20}$ & 37 \\
\hline $13_{2,11} \leftarrow 12_{1,12}$ & 6 & $17_{2,15} \leftarrow 16_{1,16}$ & 6 & $21_{2,19} \leftarrow 20_{1,20}$ & 37 \\
\hline $14_{3,12} \leftarrow 13_{0,13}$ & 6 & $18_{3,16} \leftarrow 17_{0,17}$ & 6 & & \\
\hline $14_{2,12} \leftarrow 13_{1,13}$ & 29 & $18_{2,16} \leftarrow 17_{1,17}$ & 6 & & \\
\hline $15_{3,13} \leftarrow 14_{0,14}$ & 29 & $19_{3,17} \leftarrow 18_{0,18}$ & 37 & & \\
\hline $15_{2,13} \leftarrow 14_{1,14}$ & 29 & $19_{2,17} \leftarrow 18_{1,18}$ & 37 & & \\
\hline
\end{tabular}

* 6: R. A. Toth, "Linelist of water vapor parameters from 500 to $8000 \mathrm{~cm}^{-1}$," measured values, see http://mark4sun.jpl.nasa.gov/data/spec/H $20.29:$ R. A. Toth, “Air- and $\mathrm{N}_{2}$-Broadening parameters of water vapor: 604 to $2271 \mathrm{~cm}^{-1}$," J. Mol. Spectrosc. 201, 218-243 (2000). 37: Ref. [14].

estimated from the identity between two wave functions associated with paired $\mathrm{H}_{2} \mathrm{O}$ states in the same category. As a result, there is room to adjust $j_{b d}$ slightly. In addition, with the black box theory, one focuses attentions only on the relations between the inputs and outputs and does not address what really happens inside the box. Therefore, one has to consider conclusions draw from the black box theory to be taken in a general sense. In other words, slightly different behaviors to follow the general rules among different spectroscopic parameters are well expected. It turns out that spectroscopic parameters with higher accuracies follow the rules better than those with poorer accuracies. In fact, the transition positions follow these rules the best because this parameter is completely determined by the identity and similarity of the energy levels of the $\mathrm{H}_{2} \mathrm{O}$ states without involving their wave functions. Meanwhile, in comparison with the calculated half-widths, the calculated shifts begin to follow the pair identity rule slightly later. A theoretical explanation about this is that there are larger cancelations among contributions from different correlations to the $\operatorname{Im} S_{2}\left(r_{c}\right)$ terms than those to $\operatorname{Re}_{2}\left(r_{c}\right)$. As a result, relative differences between $\operatorname{ImS}_{2}\left(r_{c}\right)$ associated with two paired lines are enhanced. The above is a brief theoretical explanation why there are delays of the pair identity rule happening in Fig. 8(c), (e), and (f).

\section{Discussions and conclusions}

As discussed in our previous paper [23], all the spectroscopic parameters of $\mathrm{H}_{2} \mathrm{O}$ lines involving high $j$ states, no matter if they are measured data or theoretically calculated values, must follow the pair identity and smooth variation rules. In addition, calculated values from any self-consistent theories should automatically follow the rules. Thus, these rules can be used to check whether the theory used in calculations contains fatal mistakes or not. Our calculated half-widths follow the rules very well as demonstrated by our previous works $[23,24]$ and meanwhile, as shown in Fig. 8, our calculated shifts follow the rules also. In contrast, some of calculated shift values in HITRAN 2008 fail to follow the rules. A question arises that how this could happen and where the mistakes could be?

We would like to note that this question differs from answering why usually the accuracy qualities of calculated shifts are poorer than those of calculated half-widths. The former is searching for mistakes made by people in their calculations and the latter is explaining different natures between the half-width and the lineshift. However, the ways to solve the problems are the same: one has to investigate the entire process within the specified formalism to figure out the answers. Because the formalism used in our calculations differ from the one used in calculating theoretical results in HITRAN 2008 [14] and because we have no access to their codes, we do not know exactly the answers at this point. But, based on our experiences, we do know where to search for them and we would like to provide some suggestions here. The first place required to check is the energy levels and wave functions of $\mathrm{H}_{2} \mathrm{O}$ states, especially those associated with high $\mathrm{j}$ values. If calculated energy levels are not accurate enough, one should replace them by more accurate ones available in literature [25]. With respect to the wave functions, one should pay attention to their symmetries associated with the four sub-blocks (i.e., $\mathrm{E}^{+}, \mathrm{E}^{-}, \mathrm{O}^{+}$, and $\mathrm{O}^{-}$) $[23,24]$ because violations of these symmetries are not allowed. In addition, we would like to note that by selecting the representation alone, the orientation of $\mathrm{H}_{2} \mathrm{O}$ in the molecular-fixed frame has not been uniquely determined. For example, in the $I R$ representation where the principle inertial axis $b$ of $\mathrm{H}_{2} \mathrm{O}$ is coincided with the $x$ axis of the molecular fixed frame, the dipole moment could still be either positive or negative because the $O$ atom can be located at either the negative or the positive sides of the $x$ axis. Therefore, one has to make sure the choices of the 0 atom location are the same both in developing the wave functions and in describing the interaction potentials of the $\mathrm{H}_{2} \mathrm{O}-\mathrm{N}_{2}$ pair. Given the fact that subroutines used in calculating the wave functions may come from other people, it becomes necessary to check these to avoid any inconsistency.

Meanwhile, one should check the resonance functions carefully. As long as potential models contain the shortrange atom-atom component, one has to evaluate two sets of resonance functions consisting of huge members. The one set used in calculating $\operatorname{ReS}_{2}\left(r_{c}\right)$ consists of resonance functions whose magnitude decrease very quickly as their arguments increase. In contrast, the other set used in calculating $\operatorname{Im} S_{2}\left(r_{c}\right)$ consists of resonance functions whose magnitudes decrease very slowly as their arguments go to infinity. This implies that one has to evaluate them over wider ranges. As a result, to evaluate the second set of the resonance functions is a big challenge. We suspect that not enough attention has been paid to the different behaviors between these two sets of 
resonance functions and that the second set has not been well depicted over their whole ranges which could introduce significant errors for calculated shifts.

Now, we summarize the salient features of the present study. By adopting the concept of the causal function from signal processing and relying on the sampling theory to replace the continuous Fourier transform by the discrete Fourier transform, we have discovered powerful tools which work excellently in evaluating the Hilbert transforms without performing the Cauchy principal integrations. With this new method, we are able to effectively and accurately calculate converged values of the $\mathrm{N}_{2}$ induced shifts of $\mathrm{H}_{2} \mathrm{O}$ lines. Thus, the challenge to calculate converged lineshifts with our formalism developed using the coordinate representation has finally been overcome. As a result, one is able to calculate both pressure broadened half-widths and pressure induced shifts to the accuracy of the approximations in the interaction-potential and trajectory models without containing convergence errors within the current frameworks of the original and the modified RB formalism.

Furthermore, after we were able to derive the Hilbert transforms, we have achieved fruitful results by finding answers to several longstanding questions. By comparing our results with those listed in HITRAN 2008, most of which are theoretically calculated values using the same potential model, one can judge how large their differences are. Then, by screening both calculated results with the two rules, one can clearly identify which ones contain fatal errors. Secondly, we are able to demonstrate significant effects on calculated shifts from the modification of the original RB formalism. Finally, we are able to verify the validity of the assumption with which one calculates the half-widths without including contributions from $\operatorname{Im} S_{2}\left(r_{c}\right)$.

The next step necessary to improve theoretically calculated results is using a properly selected set of the most accurately measured experimental values of the half-width to refine the potential parameters and then calculate calculated half-width values for comparison. Based on the new optimized potential model, one can then calculate theoretical values of half-widths and shifts for spectral lines of interest. Meanwhile, one has to keep in mind that some fundamental problems are required to address in the current RB formalism, such as how to consider the couplings between the translational and internal motions and so on. Therefore, there are new challenges ahead.

\section{Acknowledgments}

The authors dedicate this paper honoring Drs. Jean-Marie Flaud, Claud Camy-Peyret, and Alain Barbe for their invaluable contributions to the field of molecular spectroscopy and atmospheric remote sensing. The authors would like to thank Dr. C. Boulet for helpful discussion. Two of the authors ( $Q$. Ma and R.H. Tipping) acknowledge financial support from NASA under grants NNG06GB23G, NNX09AB62G, and FCCS-547. Q. Ma wishes to acknowledge financial support from the Biological and Environmental Research Program (BER), US Department of Energy, Interagency Agreement no. DE-AI02-
93ER61744 and financial support from NASA under grant NNH08ZDA001N-ACLAB. This research used resources of the National Energy Research Scientific Computing Center, which is supported by the Office of Science of the US Department of Energy under Contract no. DE-AC02-05CH11231.

\section{References}

[1] Anderson PW. Pressure broadening in the microwave and infrared regions. Phys Rev 1949;76:647.

[2] Tsao CJ, Curnutte B. Line-widths of pressure-broadened spectral lines. J Quant Spectrosc Radiat Transfer 1962;2:41

[3] Robert D, Bonamy J. Short range effects in semiclassical molecular line broadening calculations. J Phys 1979;40:923.

[4] Bonamy J, Robert D, Boulet C. Simplified models for the temperature dependence of linewidths at elevated temperatures and applications to $\mathrm{CO}$ broadened by $\mathrm{Ar}$ and $\mathrm{N}_{2}$. J Quant Spectrosc Radiat Transfer 1984;31:23.

[5] Fano U. Pressure broadening as a prototype of relaxation. Phys Rev 1963;131:259.

[6] Ma Q Tipping RH, Boulet C. Irreducible correlation functions of the $\hat{S}$ matrix in the coordinate representation: Application in calculating Lorentzian half-widths and shifts. J Chem Phys 2006;124: 014109 .

[7] Ma Q, Tipping RH, Gamache RR. Uncertainties associated with theoretically calculated $\mathrm{N}_{2}$-broadened half-widths of $\mathrm{H}_{2} \mathrm{O}$ lines. Mol Phys 2010;108:2225.

[8] Weaver HJ. Theory of discrete and continuous fourier analysis. New York: John Wiley \& Sons; 1989.

[9] Cooley JW, Tukey JW. An algorithm for the machine calculation of complex Fourier series. Math Comput 1965;19:297.

[10] Lynch R, Gamache RR, Neshyba SP. Fully complex implementation of the Robert-Bonamy formalism: Half widths and line shifts of $\mathrm{H}_{2} \mathrm{O}$ broadened by $\mathrm{N}_{2}$. J Chem Phys 1996;105:5711.

[11] Gamache RR, Fischer J. Half-widths of $\mathrm{H}_{2} \mathrm{O}, \mathrm{H}_{2}{ }^{18} \mathrm{O}, \mathrm{H}_{2}{ }^{17} \mathrm{O}, \mathrm{HD}^{16} \mathrm{O}$ and $\mathrm{D}_{2}^{16} \mathrm{O}$ : I. Comparison between isotopomers. J Quant Spectros Radiat Transfer 2003;78:289; Half-widths of $\mathrm{H}_{2}{ }^{16} \mathrm{O}, \mathrm{H}_{2}^{18} \mathrm{O}, \mathrm{H}_{2}^{17} \mathrm{O}$, $\mathrm{HD}^{16} \mathrm{O}$, and $\mathrm{D}_{2}^{16} \mathrm{O}$ : II. Comparison with measurement. J Quant Spectrosc Radiat Transfer 2003; 78:305.

[12] Gamache RR, Hartmann J-M. An intercomparison of measured pressure-broadening and pressure-shifting parameters of water vapor. J Can Chem 2004;82:1013.

[13] Gamache RR. Lineshape parameters for water vapor in the 3.2 to $17.76 \mu \mathrm{m}$ region for atmospheric applications. J Mol Spectrosc 2005;229:9.

[14] Gamache RR, Laraia AL. $\mathrm{N}_{2^{-}}, \mathrm{O}_{2^{-}}$, and air-broadened half-widths, their temperature dependence, and line shifts for the rotational band of $\mathrm{H}_{2}{ }^{16} \mathrm{O}$. J Mol Spectrosc 2009;257:116.

[15] Ma Q Tipping RH, Boulet C. Modification of the Robert-Bonamy formalism in calculating Lorentzian half-widths and shifts. J Quant Spectrosc Radiat Transfer 2007;103:588.

[16] Gray CG, Gubbins KE. In: Theory of molecular fluids. Oxford: Clarendon Press; 1984.

[17] Hahn SL. In: Hilbert transform in signal processing. Boston: Artech House; 1996.

[18] Boulet C, Robert D, Galatry L. Shifts of the vibration-rotation absorption lines of diatomic molecules perturbed by diatomic polar molecules, A theoretical analysis. J Chem Phys 1976;65:5302.

[19] Robert D, Giraud M, Galatry L. Intermolecular potentials and widths of pressure-broadened spectral lines. I. Theoretical formulation. J Chem Phys 1969;51:2192.

[20] Griem HR, Baranger M, Kolb AC, Oertel G. Stark broadening of neutral helium lines in a plasma. Phys Rev 1962;125:177.

[21] Murphy JS, Boggs JE. Collision broadening of rotational absorption lines: I. Theoretical formulation. J Chem Phys 1967;47:691.

[22] Frost BS. A theory of microwave lineshifts. J Phys B: Atom Mol Phys 1976;9:1001.

[23] Ma Q Tipping RH, Lavrentieva NN. Pair identity and smooth variation rules applicable for the spectroscopic parameters of $\mathrm{H}_{2} \mathrm{O}$ transitions involving high-J states. Mol Phys 2011;109:1925.

[24] Ma Q, Tipping RH, Lavrentieva NN. Theoretical studies of $\mathrm{N}_{2}$ broadened half-widths of $\mathrm{H}_{2} \mathrm{O}$ lines involving high J states. Mol Phys 2012, doi:10.1080/00268976.2011.646333.

[25] Barber RJ, Tennyson J, Harris GH, Tolchenov RN. A high-accuracy computed water line list. Mon Not R Astron Soc 2006;368:1087. 